hep-th/0303194

RUNHETC-2003-09

\title{
The statistics of string/M theory vacua
}

\author{
Michael R. Douglas \\ Department of Physics and Astronomy, Rutgers University, Piscataway, NJ 08855-0849 USA \\ I.H.E.S. ${ }^{\dagger}$, Le Bois-Marie, Bures-sur-Yvette, 91440 France

\begin{abstract}
We discuss systematic approaches to the classification of string/M theory vacua, and physical questions this might help us resolve. To this end, we initiate the study of ensembles of effective Lagrangians, which can be used to precisely study the predictive power of string theory, and in simple examples can lead to universality results. Using these ideas, we outline an approach to estimating the number of vacua of string/M theory which can realize the Standard Model.
\end{abstract}

March 2003

$\dagger$ Louis Michel Professor 


\section{General introduction}

String/M theory, the unified web of dual theories which subsumes superstring theory and supergravity, is by far the best candidate we have for a unified theory of fundamental physics. It describes quantum gravity, and making very simple compactifications, can lead to supersymmetric grand unified theories remarkably close to those postulated as natural extensions of the Standard Model which solve the hierarchy problem [57].

This agreement, while impressive, is still only qualitative. More precise comparison has foundered on the "vacuum selection problem." Despite the unity of the theory, string/M theory appears to describe a very large number of four dimensional (and other) vacua with inequivalent physics, most of which clearly do not describe our universe. At present we have no clue which one is relevant, or how to find it.

There are different points of view about how this problem will be solved. The simplest is to say that if we search through the possibilities, we will eventually find the right vacuum, meaning the one which agrees with our data, and we can then ignore the others. This point of view is admirably direct, and to some extent we will advocate it, but it appears that the number of vacua is so large and the problem of constructing and testing them is so complicated that one needs to better organize the problem to have any hope of success.

Many believe that rather than do an exhaustive search, we need to find a "Vacuum Selection Principle," an a priori condition which will tell us which vacuum to consider. Now at present there are no good candidates for this principle. Based on our present understanding of string/M theory, it appears that the only obvious candidate principle, nonperturbative consistency, is not very selective. One can hope that a selection principle will emerge from the study of cosmology, but it can just as well be argued that cosmology will only lead to constraints of the same general type as those we already employ in phenomenology, namely tests which must be satisfied by our vacuum or by the effective Lagrangian in some neighborhood of our vacuum in configuration space, but which do not give much a priori guidance about which vacuum to look at. Such principles are valuable but do not cut through the practical difficulties we just cited.

One can clarify many of the issues and obtain a much more well-defined problem by taking the opposite position, which is that there is no Vacuum Selection Principle in the sense we just discussed. Rather, one must simply enumerate string/M theory vacua and test each one against all constraints inferred from experiment and observation. While this task may seem like searching for a needle in a haystack, this does not make it impossible 
or uninteresting. After all, with modern technology (say a harvester equipped with a magnet) one can find needles in haystacks without much difficulty. It may turn out that upon approaching the problem systematically, we will in fact find easy ways to identify the vacua which might be relevant for our world.

In my own work, this point of view evolved in [39, 38], and led to the idea that one must get a good overall picture of how many vacua the theory has and a statistical description of their properties, to guide any such search. The present paper is an introduction to these ideas and some lines of work which this point of view has inspired, details of which will appear elsewhere 44,41].

In talking to my colleagues, I find that this point of view is sometimes considered to be defeatist, abandoning any hope of "real explanation." I believe this is not right, and to explain why I have provided a "philosophical introduction" in section 2, expanding on a discussion in [38]. Although "explanation" is a subjective concept, the most important question along these lines is whether string/M theory is falsifiable given sufficient theoretical understanding and sufficient data. In fact when one considers this point carefully, one realizes that it may not be falsifiable. The basic problem is that we have not ruled out the possibility that string/M theory contains a large or even infinite set of vacua which arbitrarily well approximate the Standard Model and any of its extensions we might hope to establish experimentally. [39] While it is reasonable to believe that this is false and that string/M theory is falsifiable, we intend to argue that this is a question which can be subject to theoretical analysis and settled, we suspect long before "the right vacuum" is identified.

To explain our point, let us imagine the logically simplest possible discussion of "string phenomenology." It would be to show that $N$ different vacua of string/M theory lead to Standard Model-like physics, but with many different values of the couplings, uniformly distributed in the space of possible couplings (we will make this more precise in section 5). Now the basic number characterizing our observational knowledge of the Standard Model is the volume in coupling space consistent with observations, measured in natural units, $O(1)$ for dimensionless couplings and $O\left(M_{p l}^{n}\right)$ for a coupling of mass dimension $n$. If we include as couplings the Higgs mass and the cosmological constant, this number is of order $10^{-120-40-10-9-9-50} \sim 10^{-238}$, where we count as independent the probability for a model to realize the observed cosmological constant, Higgs mass, fine structure constant, electron and proton mass, and a product of all other Standard Model couplings (being generous in the assumed accuracy here). This is a very high precision, but suppose string/M theory led 
to $10^{1000}$ vacua which matched the Standard Model gauge group and low energy spectrum. If so, it is likely that, in the absence of a selection principle, string/M theory would lead to no testable predictions at all.

Although this number of models may seem absurdly high, of course by multiplying a modest number of independent choices, one can easily produce much larger numbers. Furthermore, our estimate for the likelihood of matching the Standard Model is far too low, as we did not even take supersymmetry into account. In any case, at present we have no meaningful estimate of the number of vacua which might approximate the Standard Model. Indeed, we have no real argument that the number is finite; and we will argue below that if we are too inclusive in our definitions, string/M theory probably leads to an infinite number of vacua.

Thus, the primary question along these lines is to somehow estimate the number of string/M theory vacua which should approximate the Standard Model. This is obviously difficult and we will not claim to have even properly formulated the question here. What we will do is make some first steps towards properly formulating it, and try to make the case that this goal could be easier than actually finding all the vacua or even the one which describes our world. For the reasons we just discussed, it might even turn out that the answer to this question will force us to drastically re-evaluate the simple idea that "we need to find the vacuum which describes our world."

Having further justified our approach in section 2, we begin by giving a very sketchy overview of the problem of string/M theory compactification in section 3. Although necessarily somewhat simplistic, such an overview is necessary to give any content to the subsequent discussion. We also justify some of our further assumptions. First, as has been argued by many, at our present level of theoretical sophistication our only hope of making statements of the generality we need is to assume that nature has spontaneously broken supersymmetry. For reasons we discuss, we consider models which arise from type I and type II orientifold compactification on Calabi-Yau, and develop a picture based on recent work on branes and vector bundles, and on compactifications with flux.

We will also discuss some basic estimates for numbers of vacua, which can be applied even in the absence of detailed understanding of quantum corrections. In particular, one can get estimates using facts about the topology of moduli spaces, or using combinatorics of brane constructions.

An important input into any claim which uses the total number of vacua to estimate the fraction of the vacua which look like the Standard Model, is knowledge of how the 
vacua are distributed among the possibilities. We will argue that flux stabilization of moduli typically leads to "uniform" distributions.

Another point which our discussion will make, which we think will be uncontroversial, is that the problem of constructing and classifying string/M theory compactifications is very complicated. Furthermore, if one tries to summarize it in the language of effective Lagrangians, one is led to strongly suspect that the quantities which enter (superpotential and Kähler potential) are very complicated functions. Now there is a time-honored way to deal with certain types of complexity in theoretical physics. It is the statistical approach, in which we introduce ensembles of randomly chosen systems, and study expected values of the quantities of interest. The great advantage is of course that these ensembles can be far easier to formulate and study than the true system, while the hope is that some properties of the true system will hold in the average system, and will be visible in the expected values. Sometimes this approach works, and in favorable cases one even finds that some quantities of interest are universal, meaning that they do not depend on the details of the ensemble but only on a few parameters which can be determined. Clearly having such quantities would be of great value.

In section 4 , we formulate some simple ensembles of $\mathcal{N}=1$ effective supergravity Lagrangians. We also pose some questions which might be interesting to study along these lines, and might show universality. In fact, there is an ensemble which has already been studied by mathematicians (for applications to quantum chaos) which can be adapted to the problem at hand, and this will enable us to actually cite a universality result of this type, governing the distribution of supersymmetric vacua.

We regard such ensembles as tools for understanding and steps towards our primary goals of properly understanding the actual set of string/M theory vacua, and estimating the number which could describe the real world. We suggest an estimate for this number, at least from one class of construction, in section 5. Although there will be gaps in this discussion and we will not claim that our estimate is reliable, we felt this exercise was useful to illustrate the use of ensembles, and to get some preliminary sense of the problem and show which parts of it are better under control and which parts are less so. Indeed, we will not rule out the possibility that the number of vacua is large enough to spoil testability, again in the absence of other selection principles.

In section 6 , we briefly summarize and conclude. 


\section{A more philosophical introduction}

This section is an expansion on points made in 38]. It could be skipped by readers with a distaste for this sort of discussion.

As we mentioned in the introduction, there is a widespread feeling that a "theory of everything" should make unique predictions for the physics we observe. String/M theory as we understand it now does not do this, and it is this lack which is often cited as the reason why a "Vacuum Selection Principle" should exist. Of course, this argument in itself is simply wishful thinking.

Let me indulge in a little analogy. Suppose we were characters in a 1930's science fiction story, who lived on an electron orbiting a nucleus. By observation, we might discover that our particular nucleus had 9 electrons orbiting it. We might even formulate the Schrödinger equation and find that our atom was a particular solution. Even without observing other atoms, by mathematical analysis of this equation, we would discover the possibility of hydrogen, helium and so on; the amazing fact would emerge that (granting the quantization of electric charge, and taking relativistic effects into account) this equation only had about 100 solutions of the general type we observed.

Having gotten that far, we could spend a long time looking for the "fluorine selection principle" which completes the story. Of course, if the physics were really governed by the Schrödinger equation, we would never find it.

Although the analogy is a bit forced, the kernel of truth in it is that, according to

our present understanding, the consistent unification of quantum mechanics and gravity through string/M theory seems to lead to a definite set of solutions which resemble our world. This is already a great deal of predictivity, and we should see how far it can take us.

Of course, unique predictivity is not at all required for a theory to be scientifically testable and falsifiable. It is far more than we expect from most theories. Still, one hopes that a theory with "no free parameters" could do better than most theories.

The sense in which string/M theory is better than generic quantum field theory, relies on the idea that vacua are local minima, or approximate minima, of the effective potential. While all coupling constants must be vacuum expectation values of fields, since a generic effective potential in a nonsupersymmetric theory will have isolated minima, all of these expectation values will take definite values in a given vacuum. Even if the minimum is not unique, one still obtains a list of potential predictions, one for each minimum, and the theory can be falsified. 
Now the expectation that a minimum is isolated, and thus that couplings are stabilized, is a generic statement which could have exceptions. More precisely, coupling constants could in principle be time dependent, but this is unnatural (for the Standard Model couplings and especially the fine structure constant) [9]. Observation of such a variation would therefore lead to much stronger constraints on the vacuum than any mere observation of a particular fixed value, and one will have testability in the same sense. Similar comments apply to "dark energy" or "quintessence" (which is not quite as unnatural).

Of course, fitting observation provides many "vacuum selection principles" in a weaker sense. The most optimistic scenario is that future accelerator experiments will provide direct evidence for string-like or other structure which is not naturally described by four dimensional field theory, and which will give us information which will directly constrain the choice of vacuum. While this is certainly the most attractive scenario, there are many others, including the original ones described in [57], in which this appears impossible: the energy scale of the new effects is far beyond any conceivable experiment. Given that the only new energy scales we have evidence for at present are $M_{\text {Planck }} \sim 10^{19} \mathrm{GeV}$ and $M_{G U T} \sim 10^{16} \mathrm{GeV}$, these latter scenarios must be taken seriously and are perhaps even preferred.

Even without such direct evidence, fitting the known data would already be quite constraining. Besides the obvious tests of fitting the spectrum and couplings, one can propose indirect ones, for example to fit our present understanding of cosmology. An extreme example of such a test is the idea that the vacuum energy, literally defined as the value of the effective potential at its minimum, must in fact reproduce the observed cosmological constant. Other examples include the ideas that one must obtain inflation, or that uncharged scalars in a range of masses around $1 \mathrm{TeV}$ are not allowed 79 . 1 . If a unique

1 To properly discuss cosmological tests, one must grant that in early cosmology the universe does not minimize the effective potential, and in general one needs more than the low energy effective field theory. In this context, when we talk about a "vacuum," we mean not just the effective field theory at the minimum but whatever computations in the underlying theory (string/M theory) are required to make the test; of course the results of such computations will depend drastically on the choice of minimum one is working near. A simple and sometimes valid picture is that one is following some trajectory which ends up at the minimum or approximate minimum and is seeing the effective field theory along this trajectory. Such tests are appropriate within our "no Vacuum Selection Principle" assumption, but one should regard a vacuum as passing the tests if it can do so for any of the initial conditions within a chosen subset of non-zero measure. 
string/M theory vacuun (or none at all) were to pass these tests, the vacuum selection problem would be solved in a practical sense.

One difference between the principles we actually know about and the hoped-for "Vacuum Selection Principle" is that to our present understanding, no one of these tests seems more fundamental or key than the others. But the biggest practical difference between the two ideas is that the "vacuum selection principles" are a posteriori tests which require constructing and studying a vacuum in great detail.

With the present state of the art, even the basic construction and analysis of one or a few vacua is a research program requiring several man-months of effort to complete and several papers to describe. Very few vacua have been studied on even the level discussed in [57]. One can try to develop better techniques, but one should realize that the standard questions which are addressed by such analyses, motivated fairly directly by comparison with experiment, such as the structure of scales and hierarchies, the gauge group and charged matter content, indeed require at least a page to answer. One cannot hope to analyze a model in less time than it takes to read and understand the results, and the number of models is such that even this is not possible for each model.

One might at least hope that some of the tests are a priori, meaning that one can restrict attention from the start to vacua with the given property. We are certainly doing this in restricting attention to models with (according to present experiment) four dimensions. Another example is that in most perturbative constructions, the low energy gauge group is more or less fixed very early in the analysis, and it is easy to exclude models in which this is (say) too small to embed the Standard Model.

A more ambitious hope along these lines is to "engineer" vacua, taking observations as encoded in the Standard Model and its extensions and directly building models which reproduce the rough features of the observations, expecting string/ $\mathrm{M}$ theory to then tell us the fine details, such as values of couplings.

It is hard to argue against these ideas, which are good to the extent that they can be implemented and actually constrain the problem. The main problem with them is that too much of the problem seems unconstrained by observation: there are many very different ways to realize the observed matter, and many "hidden" sectors which directly influence the couplings and other data we hope to predict.

We should say a brief word about the anthropic principle here and will only say that, while interesting, we feel this is raising a different question than the one we are discussing. Surely it is true that most of the possible universes which come out of string/M theory 
do not look like four large dimensions, do not lead to macroscopic structure formation, or do not lead to environments suitable for any sort of life. Any of these conditions will also lead to tight constraints on the physical laws, and it is interesting to explore these. On the other hand, we inhabit a large four dimensional universe with specific physical laws that we determine by observation, laws surely much more specific than any anthropic consideration we can seriously study will lead to, and it is not at all clear whether any string/M theory vacuum reproduces these laws. If we know the laws, and if string theory has a precise formulation, then the question of whether string theory can reproduce the laws or not can in principle be answered without ever appealing to any of the anthropic considerations.

Having defined our problem, we can say in a nutshell the main new idea we will introduce in this work: it is to gain insight and results bearing on the problem, by studying ensembles of theories which approximate the true ensemble of vacua coming out of string/M theory. We will discuss various approximation schemes below. One general approach is to imagine the true set of vacua as a "sum of delta functions in theory space," and to approximate these delta functions with a similar but more general distribution of weights. We also make various less systematic approximations.

Although at first the distinction may seem to be splitting hairs, we stress that the ensembles we are introducing are not probability distributions but rather describe the distribution of vacua in particular regions of "theory space," without any implication that one vacuum is "more likely" than another. Such an ensemble is not normalized to unit probability; rather each vacuum contributes unit measure and the total distribution is normalized to the total number of vacua.

The main reason we emphasize this distinction is the obvious point that given that we observe one particular vacuum, the ensembles we will discuss are not directly observable. Thus certain questions which might seem to be the natural applications of an ensemble of vacua, do not really make sense. The prime example is the question "what is the probability to realize vacuum X." One might imagine pushing ideas such as the ones we discuss or related ones in quantum cosmology to the point of making statements such as "the probability of our vacuum is $10^{-47}$, while the probability of vacuum $\mathrm{Y}$ which we do not live in is $10^{-42}$ (or maybe $10^{-52}$ ), but it is not clear to us what scientific conclusions one can draw from such statements.2 In particular, even if it turned out that we live in a highly

2 This is not to say that a "wave function of the universe," which might be described by a complex-valued distribution of the type we will discuss, would be uninteresting to study. We are just not convinced that it should be used to compute relative probabilities of vacua. 
improbable vacuum according to both quantum cosmological and anthropic considerations based on string theory, we do not think this could be considered as evidence against string theory. 3 Only if the vacuum we live in is literally not a possible prediction of string/M theory, meaning either that "it does not appear on the list" or that we propose a well motivated assignment of probabilities to vacua which gives it zero probability, could we consider this as falsifying string/M theory.

Of course, there are other applications of ensembles for which the probability interpretation makes sense. For example, because of statistical uncertainty in experiment and observation, we will never get us a precise set of laws to try to make contact with, and this uncertainty could also be summarized in an ensemble of effective theories. Thus, the real problem is to see if the subset of theories which are "reasonable" fits to data contains any theories in the ensemble of string/M theory vacua.

Experimental uncertainty is important, but it should be clear that we are introducing a new type of uncertainty, more as a theoretical device, and different from experimental uncertainty. The statistics which enters in understanding experimental uncertainty is of course the bread and butter of experimental analysis and phenomenology, and a fairly mature subject. As we did in the introduction, we need to talk about experimental accuracy to make our points, and to precisely define "the accuracy to which we know the Standard Model" we would need to formalize this, but we do not need such a precise definition to make our main points here.

Finally, one idea which is certainly part of this circle of ideas is what we call the "purely statistical" scenario: that all the observed structure and couplings of the Standard Model emerge as one undistinguished choice from a completely uniform distribution of low energy theories. While in the absence of any other idea this might be attractive, for example to solve the cosmological constant problem, it may seem implausible or even repellent when applied to other aspects of physics which seem to point clearly to structure, such as the unification of gauge couplings. This may be, and our job as physicists is in

3 This is in the absence of a competing theory. Of course, if one finds a competing theory which can also explain the observations, one must judge which appears more predictive, plausible, natural, likely, or whatever. All potential competitors which we know about have far more arbitrariness than string/M theory, in particular they depend on pre-specified adjustible couplng constants, and this is already a reason to suspect they will be less predictive. One should reexamine all this if a better competitor emerges, but it seems useless to try to provide any guidance for this in advance. 
part to find structure, but it should be realized that it may simply be that string/M theory contains both vacua which realize observed physics by mechanism and vacua which realize it statistically, and this would be important to know.

We will also argue that the statistical approach is relevant for theories with mechanisms; one can try to estimate the number of grand unified models, the number of models with low energy supersymmetry, and so on. One can use ensembles to gain information about what mechanisms are easy to realize in string theory and what mechanisms are difficult, and whether the features explained by the mechanism justify the choices involved. In this sense, one could think of an ensemble which accurately represented the set of string/M theory vacua as providing a "stringy" idea of naturalness. The discussion in section 5 is intended to illustrate this idea.

Although for clarity we assume throughout this work that there is no "Vacuum Selection Principle," 6 of course we do not know whether there is one or not. Let us conclude by giving what in our opinion are the best arguments "for" and "against" a Vacuum Selection Principle.

The best hope is the esthetically motivated hope that observed physics arises from a particularly "symmetric" or "natural" string compactification. Physicists have clearly been lucky in that the fundamental laws are comprehensible at all, so why shouldn't our luck hold to the end.

Against this hope, one can make the claim that the Standard Model is actually much more complicated than one might have expected on esthetic grounds. This is a subjective feeling, and long familiarity with the Standard Model has perhaps dulled it for most of us, but this point was keenly felt by physicists of the 1930's, whose intuitions may be as valid as ours.

Still, it might be that our vacuum is at a "symmetric" point. It seems to us that this would have to mean "symmetric" in terms of how it is situated in the structure containing all the vacua, so one again needs some overall picture to make any such judgement.

The best argument we know against the idea of "Vacuum Selection Principle" is simply the following. Suppose we found a well-motivated principle $x$, other than consistency, which predicted string/M theory vacuum $X$. Suppose we then determined by observation that we actually lived in vacuum $Y$, different from $X$. Would we conclude that string/M theory is wrong? No, we would conclude that principle $x$ was wrong.

Having provided more than enough philosophy for one physics paper, let us turn to physics.

4 One might call this claim the "Ultimate Copernican Principle." 


\section{Determining the set of string theory vacua}

A huge amount of work has been done on constructing string theory compactifications, and at this point it is impossible to give a real survey. This would seem to call into question any claim that one can discuss "all" string vacua in any concrete way at all.

Our claims to this effect will rest on two general hypotheses which we feel are supported by existing work. First, because of duality, our constructions of string theory vacua are highly redundant: the known vacua can be realized by many general types of construction, and in many ways. This leads to the idea that if one could extrapolate any single class of construction out of the regime in which it is weakly coupled, one would in fact reach all vacua. Thus, if one can estimate numbers and statistics of vacua taking into account the quantum corrections, even in a qualitative way, a single class of constructions could describe a finite $O(1)$ fraction of the vacua and give a representative picture.

Second, by "all" vacua, we will mean vacua with $\mathcal{N}=1$ supersymmetry, both Minkowski and AdS. This is for the usual theoretical and phenomenological reasons, but we will suggest a further reason in section 4: by developing the ideas proposed there, information about the supersymmetric vacua could provide information about vacua which spontaneously break supersymmetry.

These hypotheses along with the relatively strong mathematical technology one can apply to this case motivate basing our considerations on constructions of $\mathcal{N}=1$ compactifications using branes in type I or type II orientifolds of Calabi-Yau threefolds. This includes $\mathrm{F}$ theory, and known dualities relate this case fairly directly to the heterotic on $\mathrm{CY}$ and (less directly) to the $\mathrm{M}$ theory on $G_{2}$ manifold constructions, to the extent that one can make a unified discussion at least of the problem without quantum corrections. The constructions differ greatly in how quantum corrections arise, but we will discuss these effects in a different way.

There are certainly constructions which have not been precisely related by duality to this class, such as asymmetric orbifolds. On the other hand, there are ideas for how to do this; for example asymmetric orbifolds have some relation to discrete torsion, and discrete torsion brings in only $O(1)$ new choices. At present it seems reasonable to think that the set of constructions we understand moderately well, or (if we are able to extrapolate to strong coupling) even the subset of type II brane constructions, describe an $O(1)$ fraction of the possibilities. 


\subsection{What is a vacuum?}

Ultimately, our goal is to characterize or count nonsupersymmetric vacua which might be candidates to describe observed physics. We are free to make various more general definitions of "vacuum" along the way, as long as we have some idea how these are related to our goal.

For us, a "vacuum" is a critical point $V^{\prime}(\phi)=0$ of the effective potential in a Lorentz symmetric four dimensional effective field theory which might express low energy predictions of string/M theory in some situation. The assumption that the problem can be discussed in effective field theory terms is motivated by the fact that so far all observed physics can be so described.

Nonsupersymmetric vacua of a theory with any reasonable effective potential will be isolated, unless the theory has a continuous global symmetry. There are arguments that string/M theory cannot have such symmetries [8], and granting this point, there appears to be no ambiguity in counting the physically relevant vacua.

The problem of counting supersymmetric vacua is somewhat more open to definition, as supersymmetric vacua can come in moduli spaces, nontrivial fixed point theories might be counted with multiplicity, and so on. To some extent there are natural ways to make these choices, based on the principle that we want a definition which depends as little as possible on the details of the effective theory, as we will discuss shortly.

The general picture the reader should keep in mind is that a moduli space of vacua in the early stages of analysis (before taking quantum corrections into account) will be assigned a number which estimates the number of vacua which will appear with all corrections taken into account. For example, one can argue very generally that the number of supersymmetric vacua in a (globally) supersymmetric sigma model with superpotential should be the Euler character of the target space, no matter what the superpotential is, because this is the value of the Witten index [90]. While one can easily find caveats and exceptions to this statement, it might still be that the Witten index is a good enough estimate of the number of vacua for our purposes.

The usual way that supersymmetric vacua are used to try to infer the properties of nonsupersymmetric vacua is to postulate a supersymmetric extension of the standard model and a hidden supersymmetry breaking sector, and treat the effects of the latter on the former as an explicit supersymmetry breaking. We will not get into this level of detail, but obviously this approach can be phrased in terms of "tests," and one can 
ask what fraction of all models contain such a hidden sector and what fraction contain a supersymmetric Standard Model which is coupled to it in the right way. What we will do instead, is to discuss an approach which could lead to "universal" predictions for the ratio of nonsupersymmetric to supersymmetric vacua, in section 4 .

Having said this, we focus on the problem of counting $\mathcal{N}=1$ four dimensional vacua with the Standard Model gauge group. We recall the standard $\mathcal{N}=1$ supergravity expression for the potential (in units $M_{p l}=1$ ) [89];

$$
V=e^{K}\left(\omega^{i \bar{j}} D_{i} W D_{\bar{j}} W^{*}-3|W|^{2}\right)+D^{2}
$$

with $K$ the Kähler potential, $\omega_{i \bar{j}}=\partial_{i} \bar{\partial}_{\bar{j}} K$ the Kähler form, $W$ a holomorphic section of a line bundle $\mathcal{L}$ with $c_{1}(\mathcal{L})=-\omega$, and $D_{i} W=\partial_{i} W+\left(\partial_{i} K\right) W$ the covariant derivative on sections of $\mathcal{L}$. $D^{2}$ represents the "D-flatness" part of the potential.

A supersymmetric vacuum satisfies $D_{i} W=0$ and has cosmological constant $\Lambda=$ $-3 e^{K}|W|^{2}$. These vacua can be Minkowski or AdS in the four dimensional space-time. Both types are equally relevant for our purposes, and we will not try to distinguish them in our counting, for several reasons.

First, from the phenomenological point of view of dynamical supersymmetry breaking, the cosmological constant will get additional corrections after supersymmetry breaking, and the only reasonable condition to enforce before taking these into account is $\Lambda>$ $-c M_{\text {susy }}^{4}$.

Second, from the theoretical point of view of inferring the distribution of nonsupersymmetric vacua from that for supersymmetric vacua, clearly we need information about all supersymmetric vacua to have any hope of doing this.

Finally, from the mathematical point of view, Minkowski vacua are much harder to count. While one has the advantage that the Kähler potential drops out of the supersymmetry conditions, which are then holomorphic, it turns out that this is far outweighed by the disadvantage that the conditions $\partial_{i} W=W=0$ are more equations than unknowns. The existence of solutions to such over-determined systems of equations is non-generic and depends on very specific features of $W$; without exact results for $W$ there is no way to decide whether such solutions exist, let alone how many might exist.

Since finding supersymmetric Minkowski vacua is not the physical problem, and it is so difficult, we will not discuss it further. Henceforth, unless otherwise specified, a supersymmetric vacuum is a solution of $D_{i} W=0$, with no constraint on $W$ or $\Lambda$, and of the D-flatness conditions. 


\subsection{Estimating numbers of vacua after quantum corrections}

Although a fair amount is known about $\mathcal{N}=1$ string/M theory compactification in the weak coupling limit, and about supersymmetric field theory at arbitrary coupling, we do not yet have a good understanding of $\mathcal{N}=1$ string/M theory at arbitrary coupling.

A lot of progress is being made on exact results, and as we discussed in 40 it seems likely to us that within a few years we will have usable exact results in string/M theory of the same character we now have for $\mathcal{N}=1$ supersymmetric gauge theory, namely a precise description of the gauge symmetry, chiral field content and superpotential for a large set of $\mathcal{N}=1$ compactifications. This would allow us to put the discussion of supersymmetric vacua on a very firm footing.

However, this may be overkill for the type of question we are asking here. We will use two simple ideas to get estimates of the number of vacua after quantum corrections.

The first idea is simply to estimate numbers of vacua at weak coupling, and use the fact that as we vary parameters, supersymmetric vacua tend to move around but are not created or destroyed. Most of our weak coupling estimates will be combinatoric, counting Calabi-Yau's, vector bundles, brane configurations etc.

Let us give as a general example of this, the problem of finding all vacua of a supersymmetric quiver gauge theory, as discussed in [37]. We consider quiver theories arising as world-volume theories of D-branes in type II strings; these have gauge group $\prod U\left(N_{i}\right)$ all matter in bifundamentals $\left(\bar{N}_{i}, N_{j}\right)$, a superpotential which is a sum of gauge invariant single trace operators, and Fayet-Iliopoulos (FI) terms. First, it can be shown that all classical supersymmetric vacua can be constructed in terms of vacua with unbroken gauge symmetry $U(1)$, by taking direct sums of the gauge groups and matter configurations. The vacua with unbroken $U(1)$ are called "simple objects" and in brane language correspond to bound states of branes. A configuration with $n$ copies of a simple object has $U(n)$ unbroken symmetry, and so on.

Let $n_{\vec{N}}=n_{N_{1}, N_{2}, \ldots}$ be the number of simple objects in the $U\left(N_{1}\right) \times \ldots$ theory (for conciseness let us denote this semisimple group as " $U(\vec{N})$ ". As a simple example, consider the " $\mathcal{N}=1^{*}$ " theory [78], a $U(N)$ theory with three adjoint chiral superfields and a superpotential $W=\operatorname{tr} X[Y, Z]+X^{2}+Y^{2}+Z^{2}$. This has the spectrum of bound states $n_{k}=1$, one for each representation of $S U(2)$.

It is easy to write a generating function for the number of all classical vacua:

$$
\sum_{\vec{N}} N_{v a c}(U(\vec{N})) q^{\vec{N}}=\prod_{\vec{N}}\left(\frac{1}{1-q^{\vec{N}}}\right)^{n_{\vec{N}}} .
$$


Suppose furthermore that these simple objects have no remaining massless matter (are "rigid"), then using the fact that pure $S U(k)$ SYM has $k$ supersymmetric vacua it is easy to obtain the generating function counting all vacua of the quantum theory. It is

$$
\sum_{\vec{N}} N_{v a c}(U(\vec{N})) q^{\vec{N}}=\prod_{\vec{N}}\left(1+\frac{q^{\vec{N}}}{\left(1-q^{\vec{N}}\right)^{2}}\right)^{n_{\vec{N}}} .
$$

For $\mathcal{N}=1^{*}$, this gives the counting $N_{\text {vac }} \sim c^{\sqrt{N}}$. As we argue below, generic brane theories have many more bound states, and the generic estimate of this type is $N_{v a c} \sim c^{N}$.

Although this was a weak coupling argument, vacua in globally supersymmetric theory will not be created or destroyed under variations of the gauge couplings or variations of the superpotential which do not change its asymptotics.

The existence of supersymmetric vacua can depend on the Fayet-Iliopoulos terms. This behavior in the classical theory is given by " $\theta$-stability" [69], according to which certain solutions of $W^{\prime}=0$ can be unstable for all values of the Fayet-Iliopoulos terms, and typical solutions are stable within some cone in the space of these parameters. One can then use (3.2) computed for some value of the FI terms, to get (3.3) at that value of the FI terms, and extrapolate the result to arbitrary coupling.

These ideas are simple but such results are not in themselves the answer, because the gauge and superpotential couplings are not parameters in string/M theory models. All of these couplings are fields, and their possible expectation values must be found by solving their equations of motion, $D W / D \phi=0$.

This is a problem, but not a disaster, because if we start at a critical point for all the other fields and follow the gradient of the superpotential, a generic non-vacuum configuration must flow either to a vacuum or to a boundary of moduli space. If one knows that one of a large class of such configurations flow to a vacuum, it is likely that most or all do. While heuristic, this idea justifies the claim that, if one sector of the theory stabilizes couplings in another sector in some generic cases, it will do so in an $O(1)$ fraction of cases.

These ideas can be made more precise by basing them on the Witten index $\operatorname{Tr}(-1)^{F}$ in supersymmetric field theory [90]. Although this is not literally the number of supersymmetric vacua, for theories with isolated vacua it is a lower bound, and probably a

5 There are exceptions to this rule which can for example lead to supersymmetry breaking in the quantum theory. Although this deserves more systematic study, we suspect this is nongeneric (fortunately, there are other ways to break supersymmetry). 
fairly accurate one in theories with generic (complicated) superpotentials and no unbroken $U(1)$ 's. There is a generalization of this index in effective supergravity theories which counts vacua with signs, as we discuss elsewhere 40,45].

Another generalization of the Witten index, to deal with unbroken $U(1)$ 's, can be motivated by returning to the quiver gauge theories. Consider theories with $U(N)$ gauge group; all of these theories have $\operatorname{Tr}(-1)^{F}=n_{N}$, because all of the vacua made up of more than one simple object have unbroken $U(1)$ 's and thus cancel out of the Witten index [90]. This is because of the pairing of the ground state with states obtained by applying the $U(1)$ gaugino operators $W_{\alpha}^{n}$.

The natural generalization of the Witten index to count these vacua as well is to count the operators in the chiral ring [20]. This number is very similar to $N_{v a c}$ but counts each unbroken $U(1)$ at low energy with multiplicity 4 ,

$$
N_{\text {chiral ring }}=N_{\text {vac }} \times 4^{N_{\text {unbroken } U(1)^{\prime} s}} .
$$

This replaces (3.3) with

$$
\sum_{\vec{N}} N_{\text {chiral ring }}(U(\vec{N})) q^{\vec{N}}=\prod_{\vec{N}}\left(\frac{1+q^{\vec{N}}}{1-q^{\vec{N}}}\right)^{2 n_{\vec{N}}} .
$$

The simpler form of this formula suggests that the number $N_{\text {chiral }}$ ring would have better formal properties than $N_{v a c}$. However our considerations below will be too crude to benefit much from this improvement.

Having seen the relevance of the Witten index and these simple generalizations of it, the second idea is to get "topological" formulas for them. Let us give an example.

For theories with compact moduli spaces of vacua, the Witten index is an estimate in the sense we want, i.e. a number which counts the likely number of vacua after further quantum corrections. This follows if we assume that quantum corrections produce a superpotential which is a generic function of the gauge invariant fields, because the Witten index will be invariant under such a deformation.

For a (non-gauge) globally supersymmetric sigma model with target space $E$, one can compute the Witten index by compactifying the Minkowski space dimensions and reducing the discussion to supersymmetric quantum mechanics. Thus, we count a moduli space $E$ with multiplicity $\chi(E)$, its Euler character. 
For (non-gauge) supersymmetric theory with a superpotential, vacua are solutions to the equations $W^{\prime}=0$. Counting solutions to systems of complex algebraic equations is a well understood problem and in a certain sense the number is topological. For example, for a generic system of $n$ independent degree $d$ polynomials in $n$ unknowns, there are $n^{d}$ simultaneous solutions. If we assumed that the $n$ equations $W^{\prime}=0$ were independent (of course they are not in general), we would get an estimate, in terms of the degree of the polynomial $W$.

Both of these considerations can be incorporated in the following formula:

$$
N_{\text {susy vac }}=\int_{\mathcal{C}} c_{n}(\Omega \mathcal{C} \otimes \mathcal{L}),
$$

where $\Omega \mathcal{C}$ is the complex cotangent bundle to the configuration space $\mathcal{C}$, and $\mathcal{L}$ is the line bundle in which the superpotential takes its values. For example, the sigma model case is $\mathcal{L}$ trivial, and this integral is the Euler number, while the degree $d$ superpotential can be treated by compactifying $\mathbb{C}^{n}$ to $\mathbb{P}^{n}$ and taking $\mathcal{L}=\mathcal{O}(d)$. This does not give $d^{n}$ but the supergravity index, which is comparable.

Heuristically, this formula is saying that to contain many vacua, a region of the configuration space must have complicated topology, large Kähler volume (in Planck units), or both.

This formula is well known to mathematicians as the general formula for the number of critical points of $W$, given that $\mathcal{C}$ is compact and $W$ is holomorphic. Although as written it assumes that $\mathcal{C}$ is a manifold, even this can be generalized [48]. In any case, it can easily be made precise if the configuration space $\mathcal{C}$ is compact and $W$ is non-singular.

Unfortunately, these conditions are almost never satisfied by explicit string/M theory superpotentials (and are literally impossible in supergravity). For noncompact $\mathcal{C}$, one can still try to use (3.5) by interpreting the integrand not as a topological class but rather as an explicit form constructed from the Kähler metric and curvature, and we will suggest some justification for this idea in section 4 . This includes the case of $W$ non-single-valued, as one can go to a covering space which makes $W$ single-valued, usually at the cost of making $\mathcal{C}$ noncompact.

Now, granting (3.5), suppose our theory is composed of two sectors $F$ and $G$, where $F$ has important supergravity corrections, while couplings in $G$ have strong dependence on fields in $F$. One can then approximate (3.5) as

$$
\begin{aligned}
N_{\text {susy vac }} & =\int_{\mathcal{C}_{F}} c_{n}\left(\Omega \mathcal{C}_{F} \otimes \mathcal{L}_{F}\right) \times \int_{\mathcal{C}_{G}} c_{n}\left(\mathcal{L}_{G}\right) \\
& \sim N_{\text {susy vac } F} \times N_{\text {susy vac } G}
\end{aligned}
$$


since the number of vacua in $G$ does not depend on the parameters.

Thus, the formula (3.5) also supports the idea that to the extent we can think of the theory as composed of two sectors, the number of vacua will be roughly the product of the numbers in each sector, even if couplings in one depend on fields in the other. We suspect that many exceptions to such claims can be constructed, but at the present state of the art it is hard to make progress without making some such claims, and we feel these arguments give some justification for them.

\subsection{Type I and type II orientifold models}

The class of models we will consider has been discussed in many works. The prototype is to compactify ten dimensional type I string theory along the same lines as the original construction of quasi-realistic $\mathcal{N}=1$ vacua due to Candelas, Horowitz, Strominger and Witten [22]. This started from the heterotic string, but worked in the large volume, weak coupling limit which is well described by $d=10, \mathcal{N}=1$ supergravity/Yang-Mills theory with the standard anomaly cancellation structure [58], and thus their general discussion applies: one compactifies to four dimensions on a Calabi-Yau threefold $M$, choosing a gauge connection for a bundle $V$ on $M$ with structure group $G \subset S O(32)$ and satisfying the anomaly cancellation condition $c_{2}(V)=c_{2}(T M)$, and the Hermitian Yang-Mills equations. One obtains an $\mathcal{N}=1$ supersymmetric low energy theory with gauge group $H$ the commutant of $G$ in $S O(32)$, and a spectrum of charged chiral multiplets coming from massless adjoint fermion zero modes on $M$.

One of the prime advantages of this construction is its relation to algebraic geometry. This is a very long story involving formidable mathematics; at this point the general classification of allowed $M$ and $V$ is not known, but there are moderately effective techniques for constructing examples and some understanding of the overall picture, which we will try to use here.

We would like to base our discussion on the following claim: there are three generalizations of this construction, which if done in full generality, and extrapolated to strong coupling, could lead to an $O(1)$ fraction of possible vacua. First, we use the equivalence between gauge field configurations and Dirichlet brane configurations, exemplified by the relation between small instantons and D5-branes [91]. This has been greatly extended and generalized, to the point where one can get a usable picture of the set of all bundles $V$. Second, one can apply "generalized T-dualities" to obtain type II orientifold constructions, 
in which perturbative gauge symmetries can come from Dirichlet branes wrapping arbitrary supersymmetric cycles. Finally, one can turn on antisymmetric gauge field strength fluxes. The claim would be that general type II backgrounds with branes and fluxes cover an $O(1)$ fraction of the possibilities.

Although a fair amount is known about branes and fluxes separately, unfortunately a complete description combining branes with fluxes is not known at present, even in the supergravity (weak coupling and large volume) limit. This is an active subject of research and the situation may improve before long. At the present state of knowledge, we are going to have to make some guesses as to how to do this.

Let us go on and discuss the choices which enter this construction.

\subsection{The choice of Calabi-Yau}

Basic introductions to Calabi-Yau compactification can be found in [57,59].

Construction of Calabi-Yau threefolds has been much studied and there is a subset which in a sense has been classified, the hypersurfaces in toric varieties. In [71], Kreutzer and Skarke classify an appropriate type of "reflexive polyhedron" which can be used to construct such a $\mathrm{CY}_{3}$, and show that the number of these is $N_{C Y_{3}}^{>}=473,800,776$. Since distinct polyhedra can lead to the same $\mathrm{CY}_{3}$, this number is an upper bound for $N_{\text {toric }} \mathrm{CY}_{3}$ (thus our notation). On the other hand, $\mathrm{CY}_{3}$ 's with distinct Betti numbers $\left(b_{1,1}, b_{2,1}\right)$ are clearly distinct; the number of distinct pairs which appear is $N_{C Y_{3}}^{<}=30,108$ which is a lower bound. There are pairs of distinct $\mathrm{CY}_{3}$ 's with the same Betti numbers, so this bound is not sharp either.

Plotting the Betti numbers produces a diagram (the "shield") which obviously has structure, supporting the idea that this is at least a natural subclass of $\mathrm{CY}_{3}$ 's. Within this class, the Euler character $\chi(M)=2\left(b_{1,1}-b_{2,1}\right)$ satisfies the bound $|\chi(M)| \leq 960$. The number of distinct $b^{1,1}$ 's for a given $\chi$ is roughly $2 \chi$ for $\chi<320$, and decreases for larger $\chi$. Some patterns in their other topological invariants are observed in [80].

Unfortunately it is not known whether all $\mathrm{CY}_{3}$ 's are of this type. Indeed, mathematicians still debate whether there are finitely many or infinitely many distinct $M$.6 Most seem to believe that the number is finite. The evidence for this, such as it is, is that (1) mathematicians know no example which is not a toric hypersurface, and (2) one can start

6 More precisely, we want the number of components of the moduli space of birational equivalence classes of complex $\mathrm{CY}_{3}$ 's. 
with an $M$ with (say) $\chi=960$ and try to increase $\chi$ by an extremal transition; so far this has not led to new examples 60].

Clearly for present purposes one can only assume that the list of [71] is representative, and use the bounds we just obtained as the estimated number of possibilities. This type of uncertainty will plague our discussion, and this estimate should be refined, but far greater uncertainties await us. From now on, when we say that "we will assume construction $X$ is representative," we mean that in the discussion in section 5 , we will assume that the choices involved at that step lead to an $O(1)$ fraction of the possibilities.

Finally, these discrete choices do not uniquely characterize the Ricci flat metric on $M$ : one has additional continuous parameters or "moduli." On a general level this is well known: there are $b_{1,1}+b_{2,1}$ moduli, each leading to a singlet chiral superfield in the low energy effective Lagrangian.

Quite a lot is known about the global structure of these moduli spaces and even explicit metrics are known, at least in the weak coupling and large volume limit. This comes from considering the related type II compactifications on $\mathrm{CY}$ with $\mathcal{N}=2, d=4$ supersymmetry, and using "special geometry." The simplest picture, with the broadest applicability, comes from considering complex structure moduli space, since there are $\mathcal{N}=$ 2 type II compactifications for which this metric is exact.

We refer to [23] for a detailed study of the "mirror quintic" $\mathrm{CY}_{3}$ complex structure moduli space, with one chiral superfield, as perhaps the simplest example with almost all the qualitative features of the general case. A simpler example with most of the features is the complex structure moduli space of the torus $T^{6}$, as discussed in many references [73, 65], while the mathematical technology for the general case is discussed in [26].

A complex torus $T^{2 n}$ can be defined as the space $\mathbb{C}^{n}$ quotiented by a $2 n$-dimensional lattice $\mathbb{Z}^{2 n}$. Explicitly, let $u^{i}$ be coordinates on the torus; we identify $u^{i} \sim u^{i}+m^{i}+Z^{i j} n_{j}$ for all $m, n \in \mathbb{Z}$, with $Z^{i j}$ a complex matrix with positive definite imaginary part; call the space of these $\mathrm{Mat}_{n}^{+}(\mathbb{C})$.

While this construction determines the complex structure on $T^{2 n}$, the relation is not one-to-one: two lattices $(1, Z)$ related by an $S L(2 n, \mathbb{Z})$ transformation lead to tori with equivalent complex structures, related by a large diffeomorphism which acts nontrivially on the periods. Thus the moduli space of complex tori $\mathcal{M}_{c}\left(T^{2 n}\right)$ is a quotient $\operatorname{Mat}_{n}^{+}(\mathbb{C}) / S L(2 n, \mathbb{Z})$.

The physical metric on this moduli space, which appears in the supergravity kinetic term, is the Weil-Peterson metric on this moduli space of flat metrics (this is a fancy way to 
say, the metric which arises from straightforward Kaluza-Klein reduction). It has Kähler potential

$$
K_{Z}=-\log \operatorname{det} \operatorname{Im} Z
$$

and constant negative curvature. Constant negative curvature is special to this example, but negative curvature is a very general feature of Weil-Peterson metrics.

One of the important qualitative features of this moduli space, and other $\mathrm{CY}_{3}$ moduli spaces, is that it has finite volume in the Kähler metric [62]. This is despite the fact that boundaries can be at infinite distance.

Finally, another important quantity which can be computed for very general $\mathrm{CY}_{3}$ 's as a function of the moduli is the vector of periods of the holomorphic three-form,

$$
\Pi^{i}=\int_{\Sigma_{i}} \Omega .
$$

Here $\Sigma_{i}$ is a basis for $H_{3}(M, \mathbb{Z})$. For $T^{6}$, for example, these are $1, Z_{i j}$, $(\operatorname{det} Z)\left(Z^{-1}\right)^{i j}$ and $(\operatorname{det} Z)$. These enter in flux superpotentials and in black hole entropy calculations, to name two applications we will call on. Note that they transform non-trivially under $S L(6, \mathbb{Z})$ and are thus not single-valued on $\mathcal{M}_{c}\left(T^{2 n}\right)$, but only on $\operatorname{Mat}_{n}^{+}(\mathbb{C})$.

\subsection{Bundle and brane configurations}

This is another long story from which we will try to extract a general picture by combining various dualities and brane arguments with considerations in algebraic geometry. We will base this on four general approaches: the "large volume" approach involving study of holomorphic bundles, the bound state/derived category approach, enumerative results on curves, and the spectral cover/T-duality approach. Our primary question is still whether the number of possibilities is finite and whether we can estimate it. We will also need to decide what fraction of constructions are likely to produce Standard Model gauge groups and chiral matter content.

In the large volume approach, the problem reduces to that of finding solutions of the hermitian Yang-Mills equations on $M$. Among the many general references on this problem are [35], [37], and [46] which will summarize some general facts relevant for superstring compactification. In particular, it is known that there is a $n$-dimensional region within the $n$-dimensional lattice of Chern classes (or brane RR charges, or K theory) for which stable bundles exist. By tensoring with a line bundle, one can always set $c_{1}(V)=0$; the region 
is then roughly characterized by the bounds $c_{2}(V)>0$ (more precisely, one has the Bogomolov bound) and bounds on the remaining invariant $c_{3}(V)$, which can only take finitely many possible values. Unfortunately no effective bound is known, but various general considerations point to a bound of the order $\left|c_{3}(V)\right|<C \cdot \chi(M)$. Furthermore, the resulting moduli spaces of bundles (of fixed topological type) are algebraic, meaning essentially that they can be defined by a finite system of equations in some (large dimensional) projective space. Although they need not be manifolds, this is good enough to believe that there will be finitely many vacua after quantum corrections.

Although not terribly concrete, these are at least good finiteness results for the large volume, weak coupling limit. Once we leave this limit, the situation is less clear.

Making a comparable discussion for type II and orientifolds requires using the general relations between vector bundles on Dirichlet branes and combinations of D-branes discussed on a basic level in [77.64]. This allows turning the problem of classifying bundles into that of understanding moduli spaces of brane configurations.

A general result which can be derived from the index theorem at large volume, but applies to all approaches, is the following. Consider a configuration of $N_{i}$ branes of type $B_{i}$ and $N_{j}$ of type $B_{j}$ in type II string theory, where each brane is "simple," i.e. comes with $U(1)$ gauge symmetry. These could be branes wrapping different cycles, or carrying different gauge connections, or whatever. In any case, the net number of chiral multiplets arising from open strings between these branes is given by a bilinear form in their RR charges (or K theory classes), the "intersection form," which we denote

$$
N_{\left(\bar{N}_{i}, N_{j}\right)}-N_{\left(\bar{N}_{j}, N_{i}\right)}=I_{i j}=-I_{j i}=\left\langle B_{i}, B_{j}\right\rangle
$$

7 For example, let us consider the dual heterotic M theory picture. Here we can add fivebranes, wrapping effective cycles $\Sigma$ satisfying $c_{2}(V)+[\Sigma]=c_{2}(T M)$. There is still an argument for finiteness of the number of solutions to this more general problem. It is that the "effective" condition in the choice of five-brane, which along with the Bogomolov inequality tend to make both bundle and brane contributions to this formula positive (physically this is to say that the branes and instantons must be BPS). the number of choices is finite here as well. However, the Bogomolov inequality only bounds a single component of $c_{2}(V)$, namely $\int_{M} c_{2}(V) \wedge \omega$, leaving open the possibility that there are infinite sequences of stable bundles with the other components of $c_{2}(V)$ running off to negative infinity. No such example is known, but it could be that they exist and are ruled out on other physical grounds (we will discuss analogous examples later). I thank Richard Thomas for a discussion on this point. 
Simple explicit formulas can be found for this form in all approaches.

There are similar formulas for type I and orientifolds [4, 27], involving the orientifold action and the class of the fixed plane. Rather than use these formulas, we are going to use a simpler description of orientifolding [43]: given a $U(N)$ quiver theory in which the intersection numbers and ranks of gauge groups have symmetry under a $\mathbb{Z}_{2}$ action on the nodes, one can restrict attention to gauge fields and matter configurations which are invariant under the symmetry (with suitably chosen signs). Some of the geometric definitions of orientifolding using "image branes" can be shown to reduce to this, and we will assume that this construction is representative, within the context we discuss below.

This allows us to find the massless matter spectrum for simple combinations of branes. However, this only scratches the surface of the problem as general holomorphic bundles correspond to general bound states of branes, typically with very complicated moduli spaces, as one would expect for classical moduli spaces of supersymmetric vacua of gauge theories with generic superpotentials. While there has been much mathematical work on the problem, it is not easy to explicitly describe the bundles and moduli spaces even on the simplest threefold, projective space $\mathbb{P}^{3}$. The Calabi-Yau case is similar but harder.

On penetrating the language and other barriers, one finds that much of this mathematics turns out to be based on ideas which have relatively simple physical translations, which we refer to as the "bound state/derived category approach," as discussed in [37]. Recent work has led to a fairly good understanding of this translation in type II theory, which in broad terms can be summarized in the claim that a supersymmetric brane configuration in type IIb at weak coupling but arbitrary Kähler moduli is a П-stable object $E$ in $D(\operatorname{Coh} M)$, the derived category of coherent sheaves. The next step in a systematic approach to the models under discussion is to classify possible orientifoldings $\Omega$; these are in a sense $\mathbb{Z}_{2}$ automorphisms of $D(\operatorname{Coh} M)$ which it is plausible to believe are obtained by conjugating the type I $\Omega$ by the action of Fourier-Mukai transforms (T-duality).

What this means in more physical terms is the following. We start with a small set or "basis" of elementary branes, at least one for each $\mathrm{K}$ theory class on $M$, and try to describe all branes as bound states of these elementary branes and their antibranes. This is done by deriving the joint world-volume theory of the collection of branes; each bound state is then a supersymmetric vacuum of this theory.

The power of this approach comes from the fact that one can find bases with simple world-volume theories. One looks for "rigid" branes, meaning those without world-volume adjoint matter, chosen so that any pair within the set has only open strings of a single 
charge (in quiver language, a single orientation of arrows), as the superpotential for up to two branes is then forced to be zero by gauge invariance, and can be computed systematically for more branes. The set of "fractional branes" in an orbifold or Gepner model [31] provides an example; one can find other bases by applying Seiberg duality to this one, and the general picture is that any bound state is a bound state of branes (not antibranes) in one of these preferred bases.

These Seiberg dualities can have various physical interpretations depending on the gauge couplings; at weak coupling one is simply using different bases of branes to describe the same bound states, while more generally couplings can flow and different dual pictures can be valid at different energy scales [19,51]. One would need to take this into account to decide which of these Seiberg dual theories are physically dual, and which are different at the scale of supersymmetry breaking (below which the duality is inoperative).

The simplest examples are the hypersurfaces in weighted projective space, a subset of the toric hypersurfaces with 7,555 elements. Some of these can be defined in string theory as Gepner models. [53] In these models, there is a preferred basis of "fractional branes," and a simple description of their intersection form. A Gepner model is essentially a $\mathbb{C}^{5} / \mathbb{Z}_{K}$ Landau-Ginzburg orbifold model, characterized by a choice of $\mathbb{Z}_{K}$ action on $\mathbb{C}_{5}$ and some continuous parameters (superpotential and FI terms). The $\mathbb{Z}_{K}$ action can be characterized by a choice of five integers $a_{i}$ which sum to $K$ and satisfy the constraint $K / a_{i} \in \mathbb{Z}$. One can compute the Betti numbers from this data, and one gets roughly $b^{1,1} \sim K$.

As discussed in [32], this model has $K$ fractional branes $B_{i}$ with $0 \leq i<K$, whose intersection form is simply expressed as

$$
\sum_{j} q^{j}\left\langle B_{i}, B_{i+j(\bmod K)}\right\rangle=\prod_{n=1}^{5}\left(1-q^{a_{n}}\right) .
$$

Enough is known about the superpotential and other data of this theory to get moduli spaces of many simple bound states, as discussed in [42]. It is known how to get similar results for general toric hypersurfaces, although this remains to be done explicitly [68].

To use such a theory in string theory, one must choose an orientifolding and enforce anomaly cancellation. The orientifoldings which are simple in the quiver language are the ones we described above which project on configurations which are invariant under a $\mathbb{Z}_{2}$ reflection of the quiver; there is a partially understood relation between this and the geometric definitions of orientifold. In any case, the resulting anomaly cancellation condition is the same as that in the large volume type I and IIb geometric orientifold 
constructions [75]. For compact CY, the fractional branes provide an overcomplete basis for the $\mathrm{K}$ theory so this determines the numbers of fractional branes up to a few adjustible parameters. These conditions can be worked out explicitly, but we will only call upon their qualitative form: in examples, the solutions have many but not all of these numbers (so, ranks of gauge group) non-zero, of order $O(10-100)$.

This of course does not mean that the ranks of gauge groups need be $O(10-100)$ as there is a lot of charged matter available to break the gauge symmetry. The basic assumption we will make in using these theories in section 5 is that one can usually (i.e., in an $O(1)$ fraction of models) use this freedom to break this to a specified subgroup while preserving supersymmetry; in brane language forming bound states between some subset of the fractional branes. If so, then an $O(1)$ fraction of models which potentially contain the Standard Model (by focusing on a subset of the anomaly cancelling branes), will contain it.

This is nontrivial and often considered the hard part of the problem; it involves details of the superpotential and D-flatness conditions and is not always true. Furthermore, even when it is true, the bound states often involve fields with string scale vevs (since this sets the scale of the FI terms), and one might worry about whether field theory is justified.

Our main reason for nevertheless making such a claim is that the relation between branes and geometric objects (bundles, objects in $D(\mathbf{C o h} M)$ etc.) relates this question to questions such as whether there is a stable bundle of the required topological type (and similar questions) for which there is independent information. As we discussed earlier, this is true for some finite region in charge space, and the anomaly cancellation conditions are usually such that $c_{2}(V)>0$, so it seems reasonable to expect an $O(1)$ fraction of solutions to the anomaly cancellation conditions to sit in this region. We should say that far more testing of this claim is possible and would be desirable.

Another relation which lends some support to this idea is the relation between brane configurations (at weak coupling) and black holes. This is obtained by reinterpreting the space-filling branes as particles in the related Ila string theory (formally, T-dualizing the Minkowski space dimensions). From the world-volume point of view, this is reducing the supersymmetric gauge theory to quantum mechanics, but many qualitative aspects such as stability and supersymmetry breaking, and the estimate $N_{v a c} \sim \chi$ (the Euler character of the moduli space), are preserved under this.

By going to the strong string coupling limit, such a brane system turns into a rather different system, a black hole. The by-now familiar idea that the entropy of a D-brane 
world-volume theories should match that of a black hole in supergravity 84 provides a very different way to get such estimates, by using the attractor mechanism in supergravity. It also provides a very different way to show that certain brane configurations can form stable bound states: if this entropy is non-zero, such a configuration must exist [73, 30]. This confirms the idea that there is a finite volume region in the charge space for which such bound states exist. 8

We have now laid out a certain style of analysis of the quiver gauge theory of fractional branes at the Gepner point, on which we will base the discussion in section 5 . These are however only a subset of the rigid branes. More rigid branes can be obtained by performing Seiberg dualities on the original quiver theory. In the well understood examples (orbifolds), all the rigid branes can be obtained this way, and we will assume these are representative. Seiberg duality acts in a relatively simple way on quiver theories with no adjoint matter 81,12,19,50,15]; one picks a node of the quiver and applies the duality of [81] to this node, treating the other gauge groups as non-dynamical. One can check that dualizing the $n$ 'th node acts on the intersection numbers as

$$
I_{i j} \rightarrow I_{i j}-2 I_{i n}-2 I_{n j}+I_{i n}\left|I_{n j}\right|
$$

This provides a large number of quiver theories from a single CY. It is not known how many are distinct; the naive estimate $2^{K}$ which comes from allowing duality on each node independently is clearly an overestimate in the known examples. There is a (still not well understood) relationship between these duality actions, the CY monodromy group (acting on Kähler moduli space) and the "phase structure" of [5], which suggests that the number should be comparable to the number of "phases" of the model, which is probably a low power of $K$. It would be nice to have similar results for the orientifolded theories, but Seiberg duality for these has not been studied systematically.

We will use these results below to estimate numbers of brane constructions which can realize the Standard Model. Let us conclude by briefly discussing the final two classes of construction. A particularly simple class of models is one in which the orientifolding fixes only curves, so that anomaly cancellation can be accomplished using only D5-branes wrapped on curves. An example is given in [1]. An advantage of this type of model is

8 One should note that the black hole entropies, which tend to go as $c^{N^{2}}$, are not in general a good estimate for $N_{v a c}=\chi$, as the black hole states are expected to contribute to $\chi$ with signs. In fact one typically obtains $\chi \sim c^{N}$. 87 
that there is a highly developed technology for counting configurations of curves on CalabiYau's, the "original" mirror symmetry technology. By wrapping branes on curves, this can be used to count numbers of vacua directly. The result can also be interpreted as an Euler character of the moduli space of curves of the given degree. This leads to an estimate of the form $c^{N}$, exponential in the charge of the branes (degree of the curves).

Finally, we should mention the spectral cover construction [34,47, which is a very powerful and general construction of bundles on elliptically fibered $\mathrm{CY}_{3}$ 's. The physical idea here is simple: by T-dualizing on the fiber, a configuration of D9-branes carrying a very general bundle can be turned into a configuration of D7 and lower dimensional branes wrapping the base. Generically, these D7-branes will sit at different places in the fibration, in which case the bundle data on these is simply the choice of a line bundle on each D7-brane. The only a priori condition on the bundles one gets out is that the T-dual of the class of the D9 on the dual fibration must be absent; even this restriction can be overcome by further generalization (physically, taking bound states of the result with D7-branes in this remaining class). Counting vacua in this type of construction thus boils down to counting the configurations of D7-branes of a particular charge.

A simpler example which is related by T-duality to the ones we discussed is to take space-filling D3-branes at points in $M$, as in [65]. If no superpotential is generated, the moduli space of $N$ such branes is obviously $M^{N} / S_{N}$. This space has Euler character roughly $(\chi(M)+N) ! / \chi(M) ! N ! \sim 4^{N}$ if $N \sim \chi(M)$, neglecting the singularities where branes coincide. Treating these singularities as quantum theories with enhanced gauge symmetry can lead to larger estimates, but still $O\left(c^{N}\right)$.

This was a long subsection, so let us recap some of the main points. First, the evidence seems consistent with the idea that there are finitely many choices at this stage, which is important, as one expects a finite fraction of these configurations to match the Standard Model. Second, a generic gauge theory with $N$ branes would be expected to contribute a $c^{N}$ multiplicity of vacua. Finally, we have systematic techniques for constructing large numbers of configurations, which we will use later to discuss the difficulty of realizing the Standard Model. 


\subsection{Flux contributions and the cosmological constant}

Besides metric, Yang-Mills and brane degrees of freedom, string/M theory contains various $p$-form gauge fields. All of the well understood compactifications can be generalized by turning on background flux for these gauge fields, as first discussed in [83 and generalized in many works (a few are [76, 13, 29]). This flux leads to a potential energy (the "flux potential") which can be explicitly computed in many examples, at least at weak coupling.

This work has led to two important physical ideas, which we will review and build upon. First, since the potential energy from the flux depends on the moduli of the internal manifold in a fairly complicated way, one expects it to have isolated minima; in other words the moduli are stabilized. This idea has a long history; recent work has focused on the use of exact results for the flux potential, and in work of Giddings, Kachru and Polchinski [54], Acharya [2] and Kachru, Kallosh, Linde and Trivedi [66], it has been shown that moduli can be stabilized at finite coupling and volume, as we discuss shortly.

Second, Bousso and Polchinski [17] have suggested that the large number of independent flux contributions can lead to a large set of vacua with a closely spaced spectrum of cosmological constants, so that it becomes likely that vacua exist with acceptably small cosmological constant. Related ideas were proposed in 49.

By the rules we stated in the introduction, this can count as a solution to the cosmological constant problem, because we are not insisting that there be a mechanism or selection principle which picks out the observed case. One still needs to check that a vacuum with the appropriate $\Lambda$ exists, is metastable, can have reasonable cosmology, and so on. Since we observe $\Lambda>0$, the constraint of metastability seems to be mild, because most likely decays are to AdS vacua, which by general considerations of quantum gravity are highly suppressed or impossible [25,7].

Thus, fluxes seem to provide concrete candidate solutions to some important problems, as well as potentially dominating the other possible types of vacuum multiplicity.

We start again with a broad outline to make some basic points. Let $C^{(p)}$ be a $p$ form gauge field, and $F^{(p+1)}=d C^{(p)}$ be its field strength. Lorentz invariance of the vacuum is preserved either by electric 4-form flux in Minkowski space, possible for $p \geq 3$ if there are $p-3$ cycles, or by taking a magnetic $p+1$-form flux in the internal space. These are interchanged under duality $* F^{(p+1)}=F^{(D-p-1)}$, so by considering both dual representations of the gauge field, we can restrict attention to magnetic fluxes. 
In a minimal energy flux configuration, $H^{(p+1)}$ is a harmonic form, and is characterized by the cohomology class of the field strength

$$
N=\frac{1}{e}\left[F^{(p+1)}\right] \in H^{(p+1)}(M, \mathbb{Z}),
$$

a $J=b_{p+1}$-component vector. For physical reasons discussed in [77, [17, the flux must satisfy a quantization condition $\int_{\Sigma} H^{(p+1)}=c N / M^{p}$; i.e. $e=c / M^{p} V_{p+1}$ in (3.11), where $V_{p+1}$ is the volume of the wrapped cycle, and $M$ a fundamental scale, typically $O\left(M_{P}\right)$ where $M_{P}$ is the higher dimensional Planck scale.

The flux contributes its potential energy to the effective potential:

$$
V_{\text {flux } 1}=\int_{M} H \wedge * H \sim M_{p l}^{2} \frac{N^{2} c^{2}}{M^{2 p} V_{p+1}^{2}},
$$

where the four dimensional Planck scale is related to the $D$ dimensional Planck scale as $M_{p l}^{2}=M_{P}^{D} V_{M}$. This formula might be modified by gravitational backreaction effects, stringy and quantum corrections.

Let us now review the discussion of Bousso and Polchinski. Following the ideas of Brown and Teitelboim [18], to get a small cosmological constant, one assumes that the effective potential is the sum of a large negative term $-\Lambda_{0}$ and the flux contribution (3.12). Although flux quantization forces (3.12) to take one of a discrete set of values, if there are enough distinct choices of flux whose energy spacings are small compared to $\Lambda_{0}$, it will be likely to find a discrete choice with cosmological constant within the experimental bound.

The necessary condition for this can be stated most simply in terms of the number distribution for vacua with a given flux potential $V$, a measure $d \mu(V)$ defined by

$$
d \mu(V)=\sum_{T \in \text { theories }} \delta(V-V(T))
$$

We will discuss this type of "ensemble observable" in more depth in section 4, but in this simple example the definition should be clear. In terms of this distribution, the condition is then simply

$$
1<<\int_{\Lambda_{0}+\Lambda_{\min }}^{\Lambda_{0}+\Lambda_{\max }} d \mu(V)
$$


where $\left(\Lambda_{\min }, \Lambda_{\max }\right)$ are the experimental bounds on the cosmological constant. Evaluating (3.13) using (3.12) gives approximately

$$
\begin{aligned}
d \mu(V) & =\sum_{N} \delta\left(V-\frac{M_{p l}^{2} N^{2}}{M^{2 p} V_{p+1}^{2}}\right) \\
& \sim \frac{\operatorname{Vol}\left(S^{J-1}\right)}{2} \int d N^{2} N^{J-2} \delta\left(V-\frac{M_{p l}^{2} N^{2}}{M^{2 p} V_{p+1}^{2}}\right) \\
& \sim \frac{\operatorname{Vol}\left(S^{J-1}\right)}{2}\left(\frac{M^{2 p} V_{p+1}^{2}}{M_{p l}^{2}}\right)^{J / 2} V^{J / 2-1} d V .
\end{aligned}
$$

Replacing the sum with an integral is reasonable when (3.14) is true, so one gets a condition

$$
1<<\Delta \Lambda\left(\frac{M^{2 p-D} V_{p+1}^{2}}{V_{M}}\right)^{J / 2} \Lambda_{0}^{J / 2-1} .
$$

One can then combine this condition with constraints on the other quantities entering (3.12) to get a picture of the class of models in which this works. First, if the geometry of the internal space is "not too anisotropic" (i.e. we are away from limits or singularities in moduli space), we can take $V_{p+1} \sim V_{M}^{(p+1) /(D-4)}$. (3.15) then reduces to

$$
\frac{\Lambda_{0}}{\Delta \Lambda}<<\left(\Lambda_{0} V_{M}^{4 / D}\right)^{J / 2}
$$

The appropriate bound on $\Lambda_{0}$ is not at all obvious. Indeed, it is not immediately apparent where negative contributions to the vacuum energy will come from, and once we find them, we will face the potential problem that we will find a series of vacua in which $-\Lambda_{0}$ can become arbitrarily negative, leading to an infinite set of vacua and complete loss of predictivity.

The simplest guess is $\Lambda_{0} \sim M_{p l}^{4}$. This works well in large extra dimension scenarios, as $V_{M}$ is large. In the traditional weakly coupled string models, with $V_{M} \sim\left(\alpha^{\prime}\right)^{D / 2}$, one finds that $\Lambda_{0} V_{M}^{4 / 6}$ is small, but since $J=b_{p+1} \sim 100$ is typical for $\mathrm{CY}_{3}$ 's, at first sight this seems viable. On the other hand, as will be clear below, the flux contributions which cancel $-\Lambda_{0}$ typically lead to supersymmetry breaking at a scale $\Lambda_{0}^{1 / 4}, \theta$, and $\Lambda_{0} \sim M_{p l}^{4}$ is not acceptable from this point of view.

In any case, this discussion demonstrates the possibility of large multiplicities of vacua with cosmological constant uniformly distributed near zero, and thus a potential solution

9 I thank Shamit Kachru for emphasizing this point. 
to this problem by our rules. The discussions in [18, 17, 49] attempted to go further and explain the observed low value as the result of a natural decay process involving nucleation of domain walls which source the flux and lower the vacuum energy, down to some minimum positive value. They found that this mechanism is difficult to realize as tunneling rates between flux vacua, even in the best case, tend to be too small. We will not insist on this or on the simple form (3.12), but only on (3.14), which could be realized by many types of degeneracy, including those in which the relevant vacua had wildly different microscopic origins.

\subsection{A finiteness conjecture}

The previous discussion was somewhat simplistic as it ignored the fact that the moduli of the internal cycles which minimize the true effective potential in fact depend on the fluxes. This was inessential to the main point of [17], but as a next step needs to be taken into account.

The most obvious question this dependence raises is that the form of (3.12) admits the possibility of sequences of vacua in which both fluxes $N$ and volumes $V_{p+1}$ run off to infinity in a correlated way, such that the cosmological constant stays finite. If so, it would simply not be true that the number of vacua is finite.

In fact the existence of infinite lists of vacua is well-known in models with more supersymmetry. The most famous example is perhaps $S^{5}$ compactification of the Ilb string, which has infinitely many vacua, parameterized by the number $N$ of quantized units of five-form flux. In this case, the radius of $S^{5}$ is proportional to $N^{1 / 4}$, so this family runs off to large volume and small cosmological constant. In [86, a similar series of $T^{6}$ flux compactifications was found. These are non-supersymmetric no scale compactifications, but there seems no reason not to expect similar supersymmetric examples.

According to our rules, this is not a problem if none of these infinite series of vacua look like the real world, and if volumes of cycles $V_{p+1}$ run off to infinity, one is certainly tempted to say that the total volume $V_{M}$ will as well, and the four dimensional Planck scale $M_{p l}$ will run off to infinity.

Thus, in the absence of further constraints, the predictivity of string/M theory depends on the conjecture that the number of consistent flux vacua with cosmological constant $|\Lambda|<\Lambda_{\max }$, a bound we choose, and compactification volume $V_{M}<V^{>}$, a upper bound, is finite. In examples (we will discuss one shortly), one also has constraints on the fluxes 
from anomaly cancellation, which depend on other input (numbers of branes and topology of the $\mathrm{CY}_{3}$ ); let us denote this input as $[B]$.

Then, the conjecture is that the total number of vacua, summing over all allowed values of the flux,

$$
N_{\text {flux vac }}\left(\Lambda_{\max }, V^{>},[B]\right) \in \mathbb{Z}
$$

is finite. We would conjecture this for any type of vacua, but supersymmetric vacua (AdS and Minkowski together) would be the simplest case to check.

This conjecture is not proven in any case we know of and might need to be further refined. One possible refinement would be to replace the bound on total volume $V_{M}$ with bounds more directly related to observation, because the appropriate bound on $V_{M}$ is very different in the traditional dynamical supersymmetry breaking scenarios, and in the "large extra dimension" scenarios. This would be worth developing, but in either case physics does place an upper bound on $V_{M}$.

From what we have said so far, the most obvious way this conjecture could fail would be to find a series of models in which $-\Lambda_{0}$, the vacuum energy at "zero flux," became arbitrarily negative, because one expects to be able to add fluxes to compensate it.

\subsection{Exact flux potential in $\mathbb{I} b$}

An exact result in the large volume limit of IIb string compactification can be obtained by writing the potential in terms of the Gukov-Vafa-Witten superpotential [61] and using the special geometry results we cited above to compute the periods of the CY. This superpotential is a function of the CY complex structure moduli $z^{i}$ and the axion-dilaton $\tau$. We will also need to discuss Kähler moduli; let $\rho$ be a Kähler modulus.

We then have

$$
W=\int \Omega \wedge\left(F_{R R}^{(3)}+\tau H_{N S}^{(3)}\right),
$$

where $F_{R R}$ and $H_{N S}$ are the Ramond-Ramond and Neveu-Schwarz three-form field strenghs of Ilb string theory. Using the quantization (3.11), this can also be written

$$
W_{f l u x}=\sum_{i} \Pi_{i}\left(N_{R R}^{i}+\tau N_{N S}^{i}\right)
$$

with $N^{i} \in \mathbb{Z}$ and $\Pi_{i}$ the periods defined above. At large volume, the Kähler potential can be obtained by $\mathrm{KK}$ reduction; it is (in terms of the $\mathrm{CY}_{3}$ moduli space Kähler potential $K_{Z}$, as in (3.7))

$$
K_{\tau, \rho}=K_{Z}-\log \operatorname{Im} \tau-3 \log \operatorname{Im} \rho .
$$


Using $K$ and $W$ in the standard $\mathcal{N}=1$ supergravity expression (3.1), one obtains the flux effective potential $V_{\text {flux } 2}$.

One cannot choose arbitrary fluxes; there is a constraint from anomaly cancellation. This will only work for $\mathrm{Ib}$ orientifolds, for the simplest case of $O 3$ planes it requires a tadpole cancellation condition of the form [54]

$$
\frac{1}{(2 \pi)^{4} \alpha^{\prime 2}} \int H_{N S}^{(3)} \wedge F_{R R}^{(3)}=\left\langle N_{N S}, N_{R R}\right\rangle=K-N_{D 3}
$$

where $\left\langle N_{R R}, N_{N S}\right\rangle$ is the intersection form, $K$ is a positive integer (the orientifold tadpole) and $N_{D 3}$ the number of space-filling D3 branes (which must be nonnegative for supersymmetry). Furthermore, one can show 61, 65 that $\mathcal{N}=1$ supersymmetry implies that this number is non-negative. This combination of facts gives a bound valid for supersymmetric vacua,

$$
0 \leq\left\langle N_{N S}, N_{R R}\right\rangle \leq K
$$

One might think that this provides an a priori bound on the total flux, which would be very helpful in proving that the number of vacua is finite. This bound may be necessary, but as we discussed above, we believe one needs to place additional conditions on the flux vacua to get a finite number. In particular, the form which appears in this bound is an indefinite form (as is any bilinear form in two independent vectors), so an infinite number of choices of flux satisfy this bound.

The formula (3.18) in itself is rather abstract; one needs to know something about the behavior of $\mathrm{CY}_{3}$ periods $\Pi^{i}$ to have any intuition for it. The basic local example is the behavior near a conifold point, at which a conjugate pair of periods behaves as

$$
\Pi_{A}=z ; \quad \Pi_{B}=\text { const }+\frac{1}{2 \pi i} z \log z+\ldots
$$

The corresponding flux superpotential is dual to the $\mathcal{N}=1 \mathrm{SYM}$ instanton superpotential [88] (we discuss this further below); in this context it was studied in [54. To get some global picture, one can consider the $T^{6}$ example, for which the periods are simply polynomial in the complex structure $Z_{i j}$, already displays a lot of structure, and we recommend that the reader unfamiliar with $\mathrm{CY}_{3}$ look at 65,86] as a start.

Let us pause to make some trivial mathematical remarks, which we find important to get the right intuition about the configuration space $\mathcal{C}$ and this superpotential, which is rather different from what intuitions based on branes or gauge theory on a compact space 
would suggest. What we have to say can be summarized mathematically as follows: $\mathrm{CY}_{3}$ complex structure moduli space, and (we conjecture) the "true" configuration space $\mathcal{C}$ with stringy and quantum corrections taken into account, is a hyperbolic space [70].

To illustrate what this means, we consider the simplest "Calabi-Yau," the elliptic curve $T^{2}$ (equivalently, we could discuss the dilaton-axion dependence in the IIb problem). Let its complex structure is $\tau$, then its periods are $m+n \tau$ for $m, n \in \mathbb{Z}$. They are not single-valued on the moduli space of complex structures, which is the fundamental region in the upper half plane, and thus the flux superpotential is not single-valued on moduli space either.

Rather, the periods are single valued on the Teichmüller space (by definition), the upper half plane $\operatorname{Im} \tau>0$. This is an open complex manifold which (for purposes of studying these periods) cannot be compactified. Physically, this is to say that since a non-zero flux breaks $S L(2, \mathbb{Z})$, there is no longer a unique large complex structure limit, but rather many such limits.

In this situation, the "topological" counting formulas we discussed earlier are not literally topological; by varying $W$ one can move critical points $D W=0$ from the upper to the lower half plane. Thus, there is no immediate estimate of the form " $N_{\text {vac }}=\chi(\mathcal{C})$ " for numbers of flux vacua. Related to this, a given period can take values in a subset of $\mathbb{C}$, and this is a possible behavior for a superpotential on $\mathcal{C}$. All this is known for $T^{2 n}$ and to some extent for $\mathrm{CY}_{3}$ moduli spaces, and we suspect it is the general picture.

Returning to more local considerations, for generic fluxes, (3.18) leads to a sufficiently complicated potential to make it very plausible that critical points are isolated in all the variables it depends on, the complex structure and axion-dilaton. Although one might worry that these critical points might be unstable to run away to weak coupling (large $\operatorname{Im} \tau$ ) or large volume $\operatorname{Im} Z$, for supersymmetric vacua this is not possible, as they necessarily have $\Lambda \leq 0$, while these limits have $\Lambda \rightarrow 0$.

Supersymmetric Minkowski vacua have been shown to exist; their physical properties are discussed in [65, 86] and many other works. However, as it stands, this effective potential has no supersymmetric AdS minima. This is because $\partial W / \partial \rho=0$ and the special form of (3.19), which forces

$$
g^{\rho \bar{\rho}} D_{\rho} W D_{\bar{\rho}} W^{*}=3|W|^{2}
$$

and thus $V_{f l u x 2} \geq 0$, with equality if $D_{i} W=0$ in the other moduli. Since $D_{i} W=0$ is as many equations as unknowns, it will have solutions; indeed this is just the problem of counting critical points where we forget about the Kähler moduli. 
This form for the potential is called "no-scale structure" and follows because this is just another way to write (3.12), with its proper dependence on moduli computed via KK reduction, and (3.12) is a positive sum of squares. The independence of $W$ on $\rho$ (which is exact in perturbation theory) also implies that this potential does not stabilize the overall volume (with more Kähler moduli, typically some but not all are stabilized).

\subsection{Violation of no-scale structure, and the origin of $\Lambda_{0}$}

No-scale structure is a feature of the large volume, weak coupling limit. In any real model, further corrections will spoil this structure and stabilize the Kähler modulus. In fact, a "no scale" nonsupersymmetric vacuum with $V=0$, if it exists in the real theory, will actually be a supersymmetric AdS vacuum.

Arguments have been made for explicit $\rho$-dependent corrections both to $K$ and to $W$. In [14], $\alpha^{4}$ corrections to the ten-dimensional IIb supergravity action were shown to produce the following correction to (3.19),

$$
\delta K_{\tau, \rho}=\frac{e^{-3 \operatorname{Im} \tau / 2}}{(\operatorname{Im} \rho)^{3}}+\ldots
$$

In [66], it was recalled that nonperturbative effects in a $U\left(N_{c}\right)$ gauge theory sector will generically lead to exponentially small corrections

$$
\delta W=M^{3} e^{2 \pi i \rho / N_{c}}+\ldots,
$$

and there are stringy nonperturbative corrections of this form as well.

Either or both corrections spoil the no-scale structure. A similar correction spoiling no-scale structure can be found in $G_{2}$ compactification, by turning on a gauge field on an ADE singularity supported on a hyperbolic 3-manifold, leading to stable AdS minima [2]. In [66] it was shown that the correction (3.21) will also lead to stable AdS minima, and that further effects can lift this to a dS minimum, in a controlled regime (weak coupling and moderately large volume).

We regard these results as valuable evidence for our basic assumption, that effective field theory can describe the physics of string/M theory vacua. However, we will have to make different arguments to claim that this stabilization works at arbitrary coupling and volume.

In the exact theory, one expects the Kähler moduli to be stabilized, simply because of genericity. In fact, the real problem is that they are overdetermined; not only do we expect 
superpotential dependence as in (3.21), but if we had gone more deeply into the D-flatness conditions in the brane sector, we would have seen that these already stabilize one (real) Kähler modulus for each homology class of simple brane in the construction (this type of argument can be found at the end of [6]), and a real treatment of this problem must bring in the D-flatness conditions.

However, to get some idea of the possibilities, let us simply assume that nonperturbative physics produces a superpotential which is the sum of (3.18) and a correction depending only on $\rho$,

$$
W=W_{f l u x}+f(\rho)
$$

We retain (3.19) for the Kähler potential. This choice was made because small corrections to $W$ can easily change the problem qualitatively (by changing the $W=0$ locus), while small corrections to $K$ generally do not.

In this case, the supersymmetry conditions for the complex structure moduli and axion-dilaton are unaffected, while the $D_{\rho}$ condition becomes

$$
0=D_{\rho} W=D_{\rho} f(\rho)-\frac{3}{\operatorname{Im} \rho} W_{f l u x}(Z, \tau)
$$

This equation determines $\rho$ in terms of $W_{\text {flux }}$ at a critical point. The resulting vacuum energy is

$$
V=-3 e^{K}\left|W_{\text {flux }}+f(\rho)\right|^{2}
$$

again implicitly a function of $W_{\text {flux }}$.

Now, the problem of finding critical points of $W_{\text {flux }}$ does not have any obvious preferred scale, and it seems likely that by varying the fluxes one can find critical points with arbitrarily large magnitude $\left|W_{\text {flux }}\right|$, even taking into account the anomaly cancellation condition (3.20) (since this is an indefinite form). In light of (3.24), this potentially violates our finiteness conjecture. There are two ways it could be saved, either by cancellations in (3.24) or by the possibility that if $\left|W_{\text {flux }}\right|$ exceeds some upper bound $W_{\max }$, the equation (3.23) will fail to have solutions. The first possibility requires an implausible conspiracy between $W_{f l u x}$ and $f(\rho)$, so we consider the second. This solution to the problem simply requires that the function

$$
(\operatorname{Im} \rho) D_{\rho} f(\rho)
$$

have an upper bound, which will be $W_{\max }$. This requires $f(\rho)$ to fall off as $\operatorname{Im} \rho \rightarrow \infty$, but we already know this is true. Requiring boundedness elsewhere more or less amounts to 
requiring that $|f(\rho)|$ itself is bounded. As we discussed, this is quite possible; indeed the function (3.21) on the upper half plane is an example.

It seems to us that some structure of this type is required to get the number of flux vacua to be finite. If we grant the simple form of the equations (3.23), then we would conclude that the most useful way to count supersymmetric flux vacua is to impose a bound

$$
e^{K}\left|W_{\text {flux }}\right|^{2}<\left|W_{\max }\right|^{2}
$$

for some $W_{\max }$, probably depending on the particular $\mathrm{CY}_{3}$ and other features of the compactification, and that the number so defined should be finite.

This would then lead to a lower bound on $\Lambda_{0}$, which in this language unfortunately depends on nonperturbative physics. It might be that the origin of $\Lambda_{0}$ and any bounds it must satisfy would be clearer in some dual picture. Anyways, we offer this as an argument for stabilization which could hold in general.

Unlike our other such arguments, the upshot of this one was not that we claim Kähler moduli are stabilized for an $O(1)$ fraction of the flux vacua. Rather, we needed to call upon properties of some (assumed) Kähler stabilization to even formulate the question of counting flux vacua. The formulation we end up with is the one given in (3.16).

\subsection{The cross-coupling problem}

The flux potential is rather complicated but at least explicit, so we can answer some interesting questions with it. One of these is whether in different vacua which might superficially agree with the Standard Model, and have acceptably small cosmological constant, the couplings are equal or at least similar, or whether they vary wildly upon varying the fluxes. On general grounds, Banks, Dine and Motl [11] suggested that the latter would be true, and more recently this has also been pointed out by Acharya [3].

For example, let us consider a class of models which all contain a common subsector of the model in which the Standard Model degrees of freedom live. For example, one can propose a configuration of branes wrapped on cycles which realize the Standard Model matter content, and which might be embedded in many different $\mathrm{CY}_{3}$ 's. This idea is sometimes called "modularity" and is certainly natural from an engineering point of view. However, the question we come to is how much of the structure of the rest of the $\mathrm{CY}_{3}$ we need to know about to predict any couplings.

We model the situation by proposing two "sectors," the "Standard Model" and everything else. We have Standard Model fields $\psi$, fields $Z$ which directly control Standard 
Model couplings, and many more fields $y$ which do not. We then postulate a superpotential of the form

$$
W=\sum N_{i} \Pi^{i}(y, z)+W_{S M}(z, \psi)
$$

Suppose we vary the fluxes $N_{i}$ by an allowed quantized amount $\Delta N$ and find a new minimum; how much do we expect the Standard Model couplings to change? To simplify the problem, we consider an infinitesminal variation $\delta N$; although this is not physical, if $W$ and $K$ are not too rapidly varying (which is generically true) $d Z / d N \Delta N$ will be approximately the same.

One's first picture is that some $N_{\alpha}$ are associated to the "cycles" which we use to build the Standard Model, while most are not, and that varying the $N_{i}$ which are not will make tiny corrections to the $Z$ 's. This can be made more precise by computing $\partial Z^{i} / \partial N^{j}$ along the minima $W^{\prime}=0$. The only general topological relation between cycles is expressed in the intersection form, $\eta_{i j}$. Thus, the type of general decoupling one might have expected would be true if

$$
\frac{\partial Z^{i}}{\partial N^{j}} \sim A_{i j} \delta_{i j}+B_{i j} \eta_{i j}+\text { small corrections. }
$$

What actually happens if we vary satisfying $\delta\left(N^{j} D_{k} W_{j}\right)=0$, is that $\delta z$ determined by

$$
\delta N^{j} D_{k} W_{j}+N^{j} \delta z^{i} D_{k} \partial_{i} W=0
$$

can be large if $D W$ is large (in the other flux directions) or $D^{2} W$ is small. Now $D_{k} W_{j} \sim \tau_{k j}$ the matrix of $U(1)$ couplings. At general points in moduli space, these will not line up with $\delta_{i j}$.

This "cross-coupling" makes it difficult to claim that quantitative aspects of one sector of the theory, such as couplings, can be independent from quantitative aspects of another. This is potentially another severe problem for the predictability of the theory, and cannot be ignored. However, it is not clear how much of a problem it is, without having some real numbers.

\subsection{Brane-flux duality}

So far we discussed brane and flux degrees of freedom separately, but it is known that this is overcounting, as many configurations have dual descriptions of both types. The prototype for this is of course the seminal work of Maldacena [72], which has been reinterpreted and generalized in many ways. For the purposes of string compactification, 
perhaps the most useful form of this duality is the "geometric duality" of Gopakumar and Vafa [56].

The simplest version of this states that a theory of $N$ D5-branes wrapped on a small $S^{2}$ in a $\mathrm{CY}_{3}$, which leads to $\mathcal{N}=1 U(N)$ super Yang-Mills theory and a quantum generated superpotential, is equivalent in a configuration with flux $N$ on a related $\mathrm{CY}_{3}$ obtained by replacing the $S^{2}$ with an $S^{3}$ (the "conifold transition"). This was proved (in a sense) in [74], and many generalizations of the basic result are known to more complicated geometries.

The full extent of brane-flux dualities is not known and we will have to make a plausible guess to deal with this. The most naive guess would be that all branes can be dualized to flux, but this is not possible as flux theories cannot have low energy non-abelian gauge symmetry, while supersymmetric gauge theories with sufficient matter (for example, $U\left(N_{c}\right)$ theory with $N_{f}>N_{c}$ flavors of matter) can. It is amusing that the supersymmetric Standard Model provides an example, though because supersymmetry must be broken at a higher scale than the strong coupling gauge scale it is not guaranteed that this has deep significance. In any case, we cannot realize the Standard Model purely within the IIb closed string sector.

A more sensible claim would be that brane theories which generate superpotentials at the quantum level (for example, $U\left(N_{c}\right)$ SYM with $N_{f}<N_{c}$ flavors of fundamental matter) can be dualized, while others can not. If so, then large numbers of brane configurations on $\mathrm{CY}_{3}$ 's are in fact redundant descriptions of the flux vacua, and should not be counted.

A test of this idea, would be to compare the numbers of supersymmetric vacua in a brane configuration on $\mathrm{CY}_{3} X$, with the number of flux vacua in the geometric dual $Y$. Since there are so many more brane configurations than $\mathrm{CY}_{3}$ 's, it is quite likely that many gauge theories are in fact dual to the same $\mathrm{CY}_{3}$ with fluxes, presumably to different vacua within the latter theory. Some field theory counterparts of this possibility have been observed in 21]. Given such an identification, reproducing the same counting of vacua on both sides would be impressive evidence for the duality.

The minimal test of this is that the number of flux vacua in the dual theory should have $c^{N}$ multiplicity (in the new sector) as we argued was generic for gauge theory. Now the dual of a theory with $N$ distinct types of (B type) brane is a $\mathrm{CY}_{3}$ with $2 N$ new classes in $H_{3}(Y, \mathbb{Z})$. We have given arguments and will give more below that this is the form we expect for multiplicities of flux vacua. 


\subsection{To be continued}

At this point we have introduced more or less all the the ingredients we will use to "count vacua" in section 5. Although we somewhat oversimplified them, we cannot do much better without a better understanding of the many open issues we mentioned (and no doubt those we didn't mention). Furthermore, our picture has been too sketchy on points such as orientifolding and anomaly cancellation, and has simply left out a great deal, such as $E_{8}$ gauge symmetry (not visible at weak coupling in $\mathrm{Ilb}$ ) and other nonperturbative light states, the brane world-volume superpotential, the detailed structure of stringy nonperturbative effects, and so on.

Nevertheless, let us conclude with a final summary of how we will combine the choices we just discussed in section 5. Of course on a basic level one picks a $\mathrm{CY}_{3}$, a brane and flux configuration and so on; but to what extent do these choices correspond to the more precise definitions of vacuum counting we gave in subsection 3.2 ?

In $\mathcal{N}=1 \mathrm{Ilb}$ theory, the chiral multiplets can be divided into the complex moduli $Z$, the dilaton-axion $\tau$, the Kähler moduli $\rho$, and the open string modes $\psi$. Techniques exist for computing classical brane world-volume theories, with a superpotential $W_{c l}(\psi, Z)$ and gauge couplings depending on $\rho$. The D-flatness conditions contain FI terms which also depend on $\rho$. In fact many $U(1)$ 's are anomalous and these couplings are partners to the anomaly cancelling couplings, lifting some of the $\rho$ 's.

In general, we can expect gauge theory sectors with small matter content (so, not including the Standard Model sector) to generate a quantum superpotential stabilizing all their fields $\psi$. On the other hand, preserving supersymmetry in the Standard Model and other sectors with large amounts of matter will tend to fix the moduli $\rho$ controlling their brane tensions (so that the different branes preserve the same $\mathcal{N}=1$ supersymmetry).

The simplest description of this physics would be to employ our conjectural braneflux duality to turn all of the branes for which quantum effects lift the moduli space, into fluxes. Thus, we will count brane configurations with moduli spaces, multiplied by flux configurations, on each relevant $\mathrm{CY}_{3}$, and justify this by appealing to (3.6).

Unfortunately, we are not keeping enough information in our considerations to decide which brane theories have quantum moduli spaces; this depends on the superpotential. We will simplistically assume that all of the hidden sector theories can have sufficiently complicated superpotentials to produce isolated vacua and make this duality appropriate. Although this is clearly not always true (for example if we realized another copy of the 
Standard Model, or a different fixed point theory), it is plausible to claim that these sectors dominate the vacuum multiplicity.

While these assumptions are clearly an oversimplification, we will still see in section 5 that interesting points will emerge from the discussion.

\subsection{The main point}

To seriously address any questions of string phenomenology, we need to make a discussion such as the one we just made, which exhibits the various choices in string theory compactification, and derives the consequences of each choice for the resulting four dimensional effective theory.

Our discussion was terribly long and technical, to the point where it is very hard to get any picture of how many possibilities will come out, and how they are distributed. And it skipped many important points; a comprehensive discussion of these models would be far longer.

This is just how string theory is at present, and string theorists must do the work to exhibit the potentially relevant vacua, to have any solid foundation for string phenomenology. Furthermore, since most of the choices and consistency conditions have little direct relation to the phenomenological considerations, it is difficult to see how to do this without listing all or at least a representative subset of the vacua.

But if listing the vacua produces an answer which is too complicated to think about, and the description of the procedure which leads to the list of vacua is too complicated to think about, then what can we do ?

\section{Ensembles of effective Lagrangians}

As one changes perspective from the problem of "finding the right vacuum" to characterizing all the vacua, one realizes that the idea of "list" in many respects gives far more information than we actually want, and is inflexible in a way which makes progress difficult. A more flexible concept might be an "ensemble" of vacua which assigns a weight to each vacuum. Armed with this concept, we might try, for example, to find a simple ensemble which approximates the true ensemble of superstring vacua well enough to address the goals we stated in the introduction.

Now a vacuum, for present purposes, is a critical point of an effective potential. Most of its structure comes from where this critical point sits in the effective theory, in the sense 
that small fluctuations around it govern the spectrum and interactions of particles. Since we need to keep so much structure of effective theory to say anything useful, we may as well change our concept to instead define an ensemble of effective theories. In words, we take a subset of the data we need to specify the effective theory: field content, potential, and other terms in the Lagrangian, and make this particular data our precise definition of "theory"; we then specify an ensemble by giving a weight function on the space of these theories. A given theory could contain any number of vacua, and the resulting ensemble of vacua is the sum of all of these vacua, each weighed by the weight of the effective field theory which includes it.

The main examples we will discuss are ensembles of $\mathcal{N}=1$ supergravity theories with chiral multiplets and no gauge multiplets. The effective potential is then determined by a choice of configuration space $\mathcal{C}$, Kähler potential $K$ and superpotential $W$. These satisfy the usual rules of supergravity [89]: in particular, the Kähler form $\omega=\partial \bar{\partial} K$ is positive definite (since it is the kinetic term for scalar fields), and the superpotential is a section of a line bundle $\mathcal{L}$ over $\mathcal{C}$ such that $c_{1}(\mathcal{L})=-\omega$.

Physically, we will think of our supergravity Lagrangian as a possible effective Lagrangian which might arise from some more fundamental theory (e.g. string/M theory), defined in the usual Wilsonian sense. First, we have implicitly chosen an energy scale $M$. All quantum effects of virtual states with energies $E>M$ are included in the effective Lagrangian. On the other hand, the Lagrangian contains all fields required to describe all particles with mass $m \leq M$ in every vacuum. It may also contain fields with $m>M$.

There is a lot to say about this dependence on scale and the role of the renormalization group in these problems, and this has been discussed in the phenomenology literature. However, for what we try to make precise in this paper, namely problems involving counting of vacua and rough estimates of likelihoods to match couplings, we do not need such a precise definition, and can think of $M$ as infinitesimal. In this case, a vacuum is defined as a critical point of the effective potential, $\partial V / \partial \phi^{i}=0$.

Thus, a "theory" for us is a triple $(\mathcal{C}, K, W)$, and the set of theories as the set of triples up to the usual geometric identifications (field redefinitions). This set has components in each of which $\mathcal{C}$ has a definite dimension, topology and complex structure. Each component is an infinite dimensional manifold, a point of which is a choice of $K$ and $W$.

One can define natural metrics and even measures on these infinite dimensional manifolds. We will not need to go far into the mathematics of this for the simple examples we give. In any case, given the ability to make such definitions, we can specify an ensemble 
of theories by giving an integrable measure on the set of theories. We will not require that it is unit normalized.

Let us give a simple example, to make this concrete. Our example will bear an obvious resemblance to the Gaussian ensembles of random matrix theory. It is a particular case of a class of ensembles studied as models of quantum chaos, as we discuss below, and of course has similarities to models commonly studied in the physics of disordered systems such as spin glasses and random potential models. Many have suggested this general analogy (e.g. see [38]), and indeed spin glasses share the $c^{N}$ multiplicity of vacua we observed in the previous discussion [85]. Below, we will add to these points of similarity, the new observation that the class of ensemble we discuss can be obtained by a simple limit of the flux superpotential.

We choose $\mathcal{C}$ out of the possibilities $\mathbb{C}, \mathbb{C}^{2}$ and so on, of arbitrary dimension $n \geq 1$, and call the $n$ chiral superfields $z_{i}$. Given a particular choice for $n$ and thus $\mathcal{C}$, we choose the Kähler potential

$$
K=\sum_{i=1}^{n}\left|z_{i}\right|^{2}
$$

and superpotential

$$
W=\sum_{\vec{I}} w_{\vec{I}} z^{\vec{I}}
$$

a polynomial of degree $d$, where $z^{\vec{I}}=z_{1}^{I_{1}} \cdots z_{n}^{I_{n}}$. Such a polynomial has $c(d, n)=(d+$ $n) ! / d ! n$ ! independent coefficients (the number of degree $d$ homogeneous polynomials in $n+1$ variables).

One ensemble (a Gaussian unitary ensemble or "GUE", since it respects $U(n)$ symmetry) could be defined by taking the coefficients $w_{\vec{I}}$ to be complex. We could define a different "GOE" ensemble by taking the coefficients real, which would be appropriate if the systems of interest had CP symmetry. In either case, we choose the coefficients with weight

$$
[d \mu(W)]=\left(\frac{\pi}{\alpha}\right)^{c(d, n)}\left[\prod_{\vec{I}} d w_{\vec{I}}\right] e^{-\alpha \sum_{\vec{I}}\left|w_{\vec{I}}\right|^{2}} .
$$

(this is unit normalized).

Finally, we can specify a weight $P_{n}$ for each of the possible dimensions $n$. If we were introducing randomness purely as a theoretical device to model generic superpotentials, we should take $P_{n}=\delta_{n, N}$, i.e. simply fix $n$ and unit normalize. On the other hand, if the 
randomness reflects a multiplicity of effective field theories arising from a more fundamental theory, we should choose the $P_{n}$ to reflect these multiplicities.

Clearly this simple example can be generalized in many ways, and we will discuss a few aspects of this below. One can also generalize it to add gauge symmetry. One would have a weight $P(G)$ for each particular choice of gauge group $G$, and randomly chosen actions by isometry of the gauge group on $\mathcal{C}$ (in the simple example of flat $K$ this is the choice of matter representation), of Fayet-Iliopoulos parameters, and of more exotic couplings.

We define the expected number of vacua, meaning critical points of $V$, in the ensemble, as

$$
\left\langle N_{v a c}\right\rangle=\sum_{\mathcal{C}} P(\mathcal{C}) \int[d \mu(K, W)] \int_{\mathcal{C}}\left[d^{2 n} z\right] \delta^{(2 n)}\left(V^{\prime}\right)\left|\operatorname{det} V^{\prime \prime}\right| .
$$

The determinant is present in order to count each isolated vacuum with weight 1 , and give a coordinate-independent result. There are some variations one can make on this which we will discuss shortly.

The first point we want to make is simply that given an ensemble, one could compute mathematically or estimate on a computer the number $\left\langle N_{v a c}\right\rangle$. We will discuss some results of this type in an extremely simplified example below, and in more examples elsewhere.

Indeed one could in principle compute the expectation value of any physical observable in a given theory in the ensemble, by integrating the observable over the ensemble. For example, one could compute the distribution of cosmological constants,

$$
\rho(\Lambda)=\frac{1}{N_{v a c}} \sum_{\mathcal{C}} P(\mathcal{C}) \int[d \mu(K, W)] \int_{\mathcal{C}}\left[d^{2 n} z\right] \delta^{(2 n)}\left(V^{\prime}\right)\left|\operatorname{det} V^{\prime \prime}\right| \delta(\Lambda-V) .
$$

The second point is that some results need not depend on the details of the ensemble. As the simplest example of this, since the number of critical points of a generic function is invariant under small perturbations of the function, the number of vacua will remain invariant if we "fuzz out" the ensemble, replacing delta function contributions to the measure with highly peaked functions of unit weight. This allows a lot of potential scope for simplifying the ensemble.

One can also entertain the hypothesis that a sufficiently complicated potential will start to look like a generic member of a simple ensemble. This is expressed more precisely in the idea of "universality," which we will return to.

Let us define some related observables which are more or less precise than (4.1). First, one can discuss the number of perturbatively stable vacua, meaning those without 
tachyons. In Minkowski space (zero cosmological constant), this means of course that the matrix $V^{\prime \prime}$ must be positive definite (it is related to the mass matrix by the positive definite metric on field space). Thus, we define

$$
\left\langle N_{\text {stablevac }}\right\rangle=\sum_{\mathcal{C}} P(\mathcal{C}) \int[d \mu(K, W)] \int_{\mathcal{C}}\left[d^{2 n} z\right] \delta^{(2 n)}\left(V^{\prime}\right)\left|\operatorname{det} V^{\prime \prime}\right| \theta\left(V^{\prime \prime}\right)
$$

where the $\theta$ function applies to each eigenvalue of $V^{\prime \prime}$.

One can go on to make the question more specific by imposing metastability with respect to tunneling (obviously this is harder to treat analytically, but some useful tricks appear in the theory of spin glasses [85]), or ask for vacua with certain qualitative properties.

One can also consider less specific questions of this type. For example, the integrand in (4.1) is not the simplest one could consider. A simpler possibility is

$$
\left\langle I_{\text {vac }}\right\rangle=\sum_{\mathcal{C}} P(\mathcal{C}) \int[d \mu(K, W)] \int_{\mathcal{C}}[d z] \delta^{(2 n)}\left(V^{\prime}\right) \operatorname{det} V^{\prime \prime}
$$

where we do not take absolute value of the determinant, and thus count vacua with signs \pm 1 depending on its sign. This type of signed measure is familiar in supersymmetry, topological field theory, and so on, and produces the supergravity index (3.5). As such, it should be much easier to compute, yet also gives information which might be useful in understanding $N_{v a c}$ or even its more specific relatives.

\subsection{Estimated number of nonsupersymmetric vacua}

To illustrate how very simple estimates of numbers of vacua can be made in this language, we consider an ensemble of globally supersymmetric theories with a Gaussian distributed superpotential, and explain how to get estimates for the density of supersymmetric vacua and nonsupersymmetric vacua.

We start with the ensemble of theories with $n$ chiral superfields $z^{i}$ taking values in $\mathbb{C}^{n}$, and a superpotential

$$
W=w+a_{i} z^{i}+b_{i j} z^{i} z^{j}+c_{i j k} z^{i} z^{j} z^{k}
$$

The $(n+1)(n+2)(n+3) / 6$ coefficients are taken from independent distributions with the Gaussian weight

$$
d \mu[W ; \alpha]=[d w d a d b d c] N(\alpha) \exp -\alpha\left(|w|^{2}+\sum_{i}\left|a_{i}\right|^{2}+\sum_{i \leq j}\left|b_{i j}\right|^{2}+\sum|c|^{2}\right)
$$


where $N(\alpha)$ is chosen to normalize the total weight,

$$
1=\int d \mu[W ; \alpha]
$$

This leads to

$$
N(\alpha)=\left(\frac{\alpha}{\pi}\right)^{(n+1)(n+2)(n+3) / 6} .
$$

Note that this ensemble is symmetric under $U(N)$ rotations $z \rightarrow U z$, but it is not translation invariant; taking $z \rightarrow z+z^{i}$ changes it. In particular, the lower moments of $W$ have a nonzero expectation value in the shifted fields. One can find ensembles with more symmetry (say $U(n+1)$ ) at the cost of changing the Kähler potential, and one might expect these to play a more important role in describing more natural starting points such as string/M theory. In any case, this ensemble is fine for purposes of illustration.

We start by considering the expected number of supersymmetric vacua. Of course we already know this for the superpotential (4.5). We need to solve the $n$ equations $W^{\prime}=0$. Each of these has degree two, and a generic system of $n$ degree two equations in $n$ unknowns will have $2^{n}$ solutions.

Let us instead do this using the formula

$$
\left\langle N_{\text {susy }}\right\rangle=\int[d \mu(W)] \int_{\mathcal{C}}[d z d \bar{z}] \delta^{(n)}(D W) \delta^{(n)}(\bar{D} W)\left|\operatorname{det} D^{2} W\right|^{2} .
$$

Since we are discussing global supersymmetry, we take $D_{i}=\partial / \partial z_{i}$ the usual coordinate derivative. It is clear at this point that the constant factor $w$ will drop out of our considerations, so we now set $w=0$ (and remove the corresponding factor from $N(\alpha)$ ).

One advantage of this approach is that we can get not just a total number but an expected density of supersymmetric vacua $d \mu_{\text {susy }}(z)$, defined by

$$
d \mu_{\text {susy }}(z)=[d z d \bar{z}] \int[d \mu(W)] \delta^{(n)}(D W(z)) \delta^{(n)}(\bar{D} W(\bar{z}))\left|\operatorname{det} D^{2} W(z)\right|^{2} .
$$

At a fixed $z$, this is a simple Gaussian integral. The integrand depends on $z$, but in a relatively simple way.

One can explicitly compute the density as a function of $z$. To make our point, we will just do it for $z=0$.

At $z=0$, we have $D_{i} W(0)=a_{i}$ and $D_{i} D_{j} W(0)=b_{i j}$. The delta functions can simply be used to set $a_{i}=0$, while the values of $c$ now drop out of the discussion. This leads to

$$
d \mu_{\text {susy }}(0)=\left(\frac{\alpha}{\pi}\right)^{n(n+3) / 2}[d z d \bar{z}] \int[d b] e^{-\alpha \sum_{i \leq j}\left|b_{i j}\right|^{2}}\left|\operatorname{det}_{i, j} b_{i j}\right|^{2}
$$


This Gaussian integral is not hard to do; the result is the probability that a randomly chosen superpotential will have a supersymmetric vacuum at the origin, or equivalently the expected number of vacua at the origin in this ensemble. It is not much harder to compute the density $d \mu(z)$, which turns out to have power law falloff.

Let us compare this to the computation of the expected density of all vacua $V^{\prime}=0$, including nonsupersymmetric vacua, evaluated at $z=0$. Starting with (4.1) with $V=$ $\sum_{i}\left|D_{i} W\right|^{2}$ and following the same approach leads to

$$
\begin{aligned}
& d \mu_{v a c}(0)=\left(\frac{\alpha}{\pi}\right)^{(n+1)(n+2)(n+3) / 6-1}[d z d \bar{z}] \\
& \int[d a d b d c] e^{-\alpha \sum|a|^{2}+\sum|b|^{2}+\sum|c|^{2}} \delta^{(n)}\left(b_{i j} a_{j}^{*}\right) \delta^{(n)}\left(b_{i j}^{*} a_{j}\right)|\operatorname{det} M|
\end{aligned}
$$

where $M$ is the matrix of second derivatives $V^{\prime \prime}$, a $2 n \times 2 n$ hermitian matrix

$$
M_{i, j}=\left(\begin{array}{cc}
b_{i k} b_{k j}^{*} & c_{i j k} a_{k}^{*} \\
c_{i j k}^{*} a_{k} & b_{i k} b_{k j}^{*}
\end{array}\right) .
$$

One then solves the $\delta$ function constraints for $b$, since in a nonsupersymmetric vacuum $a \neq 0$, and proceeds above to obtain a density, which could be integrated to obtain the total expected number of nonsupersymmetric critical points. Such results and the more interesting expected number of stable nonsupersymmetric vacua will be discussed in [44,41].

\subsection{Universal results}

The first question one should ask about the type of result we just described is to what extent they are particular to a specific ensemble, and to what extent they reflect properties shared by many ensembles and which it is reasonable to believe are shared by the ensemble of string/M theory vacua.

In general, results do depend on the specific choice of ensemble. In (4.6), this includes the choice of equal variances for $a, b$ and $c$; clearly the choice of distribution which weighs discrete factors such as gauge group and matter content will be even more important. Claiming that a specific choice reproduces some aspect of the ensemble of string/M theory vacua is therefore non-trivial. Thus, a good answer to this question requires some ability to work with the string/M theory ensemble, and this is why the simpler considerations of this section would have little content without the more complex discussion of section 3 .

However, the most interesting and potentially useful questions would be those whose answers display universality. There are various ways one can try to make this concept 
precise, but one typical and standard notion is to consider a family of ensembles parameterized by an integer $N$, and claim that the $N \rightarrow \infty$ limit is a universal function of a few parameters which can be extracted from the ensemble, while $1 / N$ corrections to the leading behavior may be less universal.

In work of Bleher, Shiffman, and Zelditch on quantum chaos ([16,92], and see also the references there); such a universal limit is discussed for essentially the ensemble we just discussed. One considers a compact Kähler configuration space $\mathcal{C}$, and a positive line bundle $\mathcal{L}$. One then considers a family of Gaussian distributed holomorphic sections $W_{N} \in \mathcal{L}^{N}$ with the measure (here $\left(\operatorname{vol}_{\omega}\right)=\omega^{n} / n$ ! is the volume form):

$$
d \mu[W]=\exp -\int_{\mathcal{C}}\left(\operatorname{vol}_{\omega}\right) e^{N \cdot K}|W|^{2},
$$

and considers average properties of the critical points of $W$.

As $N \rightarrow \infty$, using the simple estimates we discussed earlier, the number of critical points will grow as $N^{n}$ (for $n$-dimensional $\mathcal{C}$ ), and thus one expects their average spacing to go as $N^{-1 / 2}$. In the limit, the distribution of critical points becomes universal:

$$
d \mu[W]=\left(\operatorname{vol}_{\omega}\right) \times c_{n} N^{n}\left(1+\mathcal{O}\left(\frac{1}{d}\right)\right)
$$

with $c_{n}$ a universal dimension dependent constant.

These results are essentially local and one might expect them to hold for suitable sums over sections even in the case of more physical interest, in which $\mathcal{C}$ is noncompact. This supports our earlier formula (3.5) as it gives a limit of the problem in which this formula becomes exact.

If one focuses on the structure at the scale of the average spacing between vacua by defining $Z=N^{-1 / 2} z$, one finds in addition that correlation functions involving the products of densities of vacua at distinct points become universal. In this sense, the local structure of the effective field theory becomes universal. Physical applications of such results might include computing the probability that another nearby vacuum could destabilize the vacuum of interest, or that flow from one critical point to another realize sufficient inflation. Such questions will be studied in [44] and future work; these are questions which one has little hope of addressing at present except in ensembles.

Finally, the parameters which entered into this example (derived from the Kähler metric and parameters of the distribution) will control the expected number of nonsupersymmetric vacua as well. This is the sense in which we would claim that general information about supersymmetric vacua can determine numbers of nonsupersymmetric vacua, which we will explore in 44,41]. 


\subsection{The ensemble of string/M theory vacua}

Besides explicitly defining ensembles, one can implicitly define ensembles. Obviously the primary one we are interested in would be an "ensemble of all theories coming out of string/M theory." This ensemble is not computable at present, but perhaps if we can precisely define it, some approximation to it will someday be computable.

The basic idea one wants to implement is the following. In section 3 we discussed the many, many choices which enter into the construction of a compactification in string/M theory. As is much discussed in the literature, each of these choices leads to a low energy effective Lagrangian, with specific field content and couplings, valid in a certain region of configuration space. We want to define an ensemble for which the measure which is a sum of delta functions, one for each effective field theory which is obtained from a given choice of the discrete compactification parameters.

Each effective field theory in the ensemble so defined will in general describe many vacua. Although so far we have stressed the idea that the ensemble will contain distinct effective field theories each corresponding to one choice of the compactification data, we could also imagine that a given compactification is not described by a single effective theory but rather by a collection of "dual descriptions," each with partially overlapping regions of validity. To do this well, we must generalize our definition of ensemble, for the following reasons.

First, we may only trust a given effective field theory if the fields live in a certain region of configuration space. This is definitely not a problem of principle and there is no a priori restriction on the region which can be used. In particular, there is no reason that one effective field theory cannot be valid over a range of configurations with relative distance large compared to $M$, or even large in Planck units. However, it often does happen that a specific derivation breaks down in such circumstances (typically because new light states come down), so one needs to allow for this type of partial information. Now there is a way to deal with it given our previous definitions: we simply take $\mathcal{C}$ to be a manifold with boundary, which cuts out the regions we do not trust. However, this will lead to many complicated regions $\mathcal{C}$ and a complicated description. We would prefer to describe this information more simply.

Second, different effective field theories can be dual, and describe the same physics. While the observables that we compute should reflect these identifications, this does not immediately bear on the question of whether we count two dual vacua in dual effective field 
theories as equivalent or not. If they do not come from dual underlying compactifications, they clearly count as distinct possible vacua in any reasonable theoretical formulation. Since we already have weights, there is no difficulty in representing this.

Third, it may be that two choices of the discrete compactification data are dual and lead to the same set of vacua. It could even be that two choices are only partially dual: that there is a subset of vacua of theory A which are to be identified with a subset of vacua in theory B, while the other vacua are distinct (or perhaps identified with other theories). This leads to problems of description analogous to the first point above.

All these features lead to complexity in the relation between the starting point (string/M theory) and the final ensemble, but they need not imply inherent complexity in either the starting point or the final ensemble. Nevertheless we need some formal language to describe it.

The suggestion we will make to treat it is the following. We generalize our weight $P_{n}$ which represented the weight of a given configuration space $\mathcal{C}$, to a weight function on $\mathcal{C}$, a real function $\rho(\mathcal{C} ; z, \bar{z})$. And we generalize the definition of the expected number of vacua to

$$
\left\langle N_{v a c}\right\rangle=\sum_{\mathcal{C}} \int[d \mu(K, W)] \int_{\mathcal{C}} \delta^{(2 n)}\left(V^{\prime}\right)\left|\operatorname{det} V^{\prime \prime}\right| \rho(\mathcal{C} ; z, \bar{z}) .
$$

This is a rather broad generalization which certainly allows the freedom to deal with the problems we just discussed. If we only trust a given effective field theory to describe a subset of configuration space, we set $\rho=0$ outside that subset. If we have two dual theories which each describe a region $R$, we set $\rho=\frac{1}{2}$ for each of the theories within $R$, and so on.

Obviously this is a highly redundant and ambiguous description, and it would be implausible to claim that string/M theory leads to particular preferred $\rho$ 's, $\mathcal{C}$ 's, and so forth. On the other hand, to the extent that string/M theory leads to many different effective theories, it is not a priori implausible to claim that the sum of the contributions to the ensemble from many unrelated effective theories produces a much simpler ensemble, with simple choices for $\rho$ along with the rest of the data, than any of the individual effective theories might suggest. Such a claim should be evaluated by comparing results computed in a given ensemble to results derived directly from sets of many actual compactifications of string theory, in a spirit somewhat analogous to the original tests of superstring duality.

We are not claiming that all physical questions about string/M theory can be usefully addressed this way. But to answer some questions, starting with the number of vacua, and going all the way to our primary question of how many vacua well approximate the 
Standard Model, we do not need to reproduce the stringy ensemble precisely. To the extent that string/M theory has finitely many vacua (with "reasonableness conditions" of the type we suggested in section 3), the true ensemble will always look like a sum of delta functions, and indeed this is where the predictive power of string theory lies. On the other hand, we might well get good estimates for the quantities we have stressed as potentially accessible to this approach by using an ensemble with a smooth measure on theory space, which could be far simpler than the true ensemble coming from string theory.

We conclude by stressing the importance of the claim that this "true ensemble of string/M theory effective field theories" exists and is well defined, up to ambiguities in describing dual sets of theories by specific choices of $\rho$. The existence of this ensemble depends on only two essential points. First, we need a precise definition of string/M theory, in which vacua are described by specific effective field theories. Second, we need "local finiteness" of the ensemble, meaning that there should not be an infinite number of distinct (non-dual) compactifications which lead to the same or arbitrarily similar effective field theories. One of the main points of the discussion in section 3 was to give arguments that the number of physically relevant vacua is finite, which would certainly imply local finiteness.

Granting that this ensemble exists, to some extent it and approximations to it can play the role for $\mathcal{N}=1$ and nonsupersymmetric theories, which moduli spaces of vacua played for studying theories with extended supersymmetry. The subsequent examples will illustrate this point.

\subsection{The flux superpotential ensemble}

We can define the ensemble of flux superpotential vacua as follows. We take $K$ to be the standard Kähler metric. And we take $W$ distributed as

$$
d \mu(W)=\sum_{N \in \mathbb{Z}} \delta\left(W-N_{i} \Pi^{i}(z)\right) e^{-\alpha_{i j} N^{i} N^{j}}
$$

The exponential factor could be used to get convergence of the sum, and a finite number of vacua, and also to enforce the $\eta_{i j} N^{i} N^{j}$ anomaly cancellation constraint, by coupling this to a parameter and taking an integral transform. The other constraints we discussed in section 3 enter in different ways: the cosmological constant is an observable, while excluding the large volume limit would require cutting this region out of the complex 
structure moduli space, and exclusion of the large Kähler modulus limit (in Ilb) would enter into the discussion in subsection 3.9.

The relation between this ensemble and the Gaussian orthogonal ensemble we discussed in the previous section should be clear: we get the latter from the former by forgetting about the quantization condition on the $N^{i}$. In other words, we "fuzz out" the delta functions.

The main point we take from this is that simple Gaussian ensembles such as those studied by [16], are actually quite similar to the actual ensemble of flux superpotentials, lending support to the idea that they can well approximate their physics. We discuss this further in [44].

One can take the same type of $d \rightarrow \infty$ limit we discussed above, by considering $K \rightarrow d K$ and superpotentials of the form

$$
W=\sum_{N} N_{i_{1} \ldots i_{d}} \Pi^{i_{1}} \cdots \Pi^{i_{d}}
$$

Since $K$ always enters as $K / M_{p l}^{2}$, the scaling $K \rightarrow d K$ corresponds physically to "taking $M_{p l} \rightarrow 0$ " or in other words considering a series of problems in which the effects of supergravity (compared to global supersymmetry) become increasingly important. In any case, the limit distribution is (plausibly) (4.11); the vacua become uniformly distributed with respect to the volume form derived from the Kähler form.

Since the fields entering into this superpotential will control coupling constants in the observable sector, this claim gives some precise meaning to the idea that "flux vacua are uniformly distributed in the space of couplings." Uniform here means with respect to the Kähler metric on moduli space.

\subsection{Uniform ensembles of effective $\mathcal{N}=1$ gauge theories}

Here we discuss simple ensembles of low energy effective theories, which are not directly motivated by string/M theory. These are important because they are the simplest possible guess as to what will come out. Much more thought should be given to what would be useful here, taking into account the RG and the eventual phenomenological tests.

Let us just give two very simple examples. The first is a simple expression of the traditional idea of naturalness, motivated by perturbative renormalization group considerations. The second would be appropriate for a theory with a duality symmetry. 
We fix the Kähler potential (say it is canonical), and fix a gauge group $G$, and matter with a linear gauge action in representation $R$. We enumerate all gauge-invariant couplings $g_{k} z^{k}$, where $g_{k}$ has canonical mass dimension $3-k$.

An ensemble is then specified by a distribution for these couplings. Of course, we cannot simply integrate over all couplings with Lebesgue measure, as this distribution is not normalizable.

An obvious requirement to impose is that the couplings be natural with respect to a UV scale $M$; in other words $g_{k} M^{k-3}$ should be $O(1)$. Let us also ask that dimensionless couplings are $O(1)$.

We choose a positive number $\alpha$, and take couplings with weight

$$
d \mu[W]=\exp -\alpha M^{6+n} \int_{B_{M}}|W(z)|^{2} d^{n} z,
$$

where $B_{M}$ is the ball $\sum\left|z^{i}\right|^{2} \leq M^{2}$, with the volume form derived from the Kähler metric. This more or less says that the sum of dimensionless couplings squared, measured in units of the cutoff, is at most $1 / \alpha$.

The Gaussian form is a choice of course; one could also bound the integral of $|W|^{2}$ above, or try other weights. It would be interesting to decide which choice best reflects traditional ideas of naturalness.

One might also try to fix the arbitrary choice of $\alpha$ by taking $\alpha=1$, motivated by the idea that a theory with coupling $g>1$ should be dual to another theory with coupling $1 / g$.

To better implement this idea, let us define the $(G, \Gamma)$-uniform ensemble, appropriate to a theory with duality symmetry. Suppose we know a theory admits $\Gamma$ duality symmetry, where $\Gamma$ is a discrete group. Typically, the duality group $\Gamma$ is a discrete subgroup of some continuous group, $G$, with a natural action on the couplings. In this case, the natural or " $(G, \Gamma)$-uniform" ensemble (or, if there is an obvious candidate $G$, the $\Gamma$-uniform ensemble), uses a measure with $G$ symmetry, and integrates over a fundamental region of $\Gamma$.

For example, the natural ensemble of $\mathcal{N}=4$ supersymmetric gauge theories according to this criterion is $S L(2, \mathbb{Z})$-uniform, where the complex gauge couplings $\tau=\theta / 2 \pi+8 \pi i / g^{2}$ are distributed according to the measure $d \tau /(\operatorname{Im} \tau)^{2}$, over a fundamental region of $S L(2, \mathbb{Z})$. 


\subsection{Ensembles of quiver gauge theories}

The minimal information in an ensemble of gauge theories is a distribution $d \mu(G, R)$ over the choice of gauge group and representation. In the case of $U(N)$ quiver gauge theories, this data is very simple: the gauge group is a product $\prod U\left(N_{i}\right)$ and thus specified by a list of non-negative integers $N_{i}$ with $1 \leq i \leq K$, while the matter content is specified by the $K \times K$ matrix $C_{i j}$ of multiplicities of chiral multiplets in the $\left(\bar{N}_{i}, N_{j}\right)$. This will be the intersection matrix $I_{i j}$ of (3.9) plus a symmetric matrix if non-chiral matter is also present, which is generically not expected.

Thus, an ensemble of quiver gauge theories will be specified by a distribution $d \mu\left(N_{i} ; I_{i j}\right)$. This could also depend on additional information such as couplings, but we will not consider this.

We can now ask the question: if we consider the collection of all $\mathrm{CY}_{3}$ 's (that we know about) and all quiver gauge theories which arise as world-volume theories of type II branes, what ensemble do we get? Since we have techniques for computing these gauge

theories, this question could be studied by various means, for example by Monte Carlo (i.e. generating examples by computer).

We first consider a toy example which can be solved, and use it to motivate some simple model ensembles which illustrate some likely features of the true ensemble. The toy example is the ensemble of supersymmetric theories which describes bound states of two elementary branes $B_{1}$ and $B_{2}$, with $k$ bifundamentals in the $\left(\bar{N}_{1}, N_{2}\right)$. As discussed in [36, 37, the spectrum of bound states (simple objects) in this theory is easy to describe completely. Assuming a positive FI term, if and only if the dimension of the gauge group minus the number of chiral matter fields is 1 , then there will be a unique supersymmetric configuration (up to gauge equivalence) breaking to $U(1)$, i.e. a simple object. Thus, there is a unique bound state $N_{1} B_{1}+N_{2} B_{2}$ for each pair $\left(N_{1}, N_{2}\right)$ satisfying

$$
N_{1}^{2}+N_{2}^{2}-k N_{1} N_{2}=1 .
$$

The net number of chiral multiplets between two such simple objects $B^{\prime}$ and $B^{\prime \prime}$ with charges $\left(N_{1}^{\prime}, N_{2}^{\prime}\right)$ and $\left(N_{1}^{\prime \prime}, N_{2}^{\prime \prime}\right)$ is the intersection number, $\left\langle B^{\prime}, B^{\prime \prime}\right\rangle=N_{1}^{\prime} N_{2}-N_{2}^{\prime} N_{1}$. Thus a supersymmetric brane theory made up of such bound states is specified by a list of simple objects with multiplicity; the spectrum of chiral multiplets is determined by the intersection numbers, and the resulting theory will have no superpotential. 
Furthermore, this spectrum satisfies a symmetry under a simplified form of Seiberg duality (the reflection functor), which acts as $\left(N_{1}, N_{2}\right) \rightarrow\left(k N_{1}-N_{2}, N_{1}\right)$. In fact, the charges of simple objects are real roots of the Kac-Moody algebra with generalized Cartan matrix $\left(\begin{array}{cc}2 & -k \\ -k & 2\end{array}\right)$, and these transformations act on the charge vectors as Weyl reflections. All simple objects can be obtained from one of them by a sequence of Weyl reflections, and this leads to a set parameterized by a single integer $n$. Let $B_{n}$ with $n \leq 0$ be the sequence $\left[B_{0}\right]=(k, 1),\left[B_{-1}\right]=\left(k^{2}-1, k\right)$, etc. and $B_{n}$ with $n>2$ be the sequence $\left[B_{3}\right]=(1, k)$, etc.

The simplest resulting world-volume theories are those describing combinations of two simple objects $B_{a}$ and $B_{b}$ (which is enough to get all others as bound states). The Weyl reflection symmetry can be used to take $B_{a}=B_{1}$; then if $\left[B_{b}\right]=\left(N_{1}^{\prime}, N_{2}^{\prime}\right)$, the intersection number will be $N_{2}^{\prime}$, and the resulting collection of quiver theories will be the set of $U\left(N_{a}\right) \times U\left(N_{b}\right)$ theories with intersection number $I_{a b}=N_{2}^{\prime}$. Thus the corresponding distribution is precisely

$$
d \mu\left(I_{a b}\right)=\sum_{n \neq 1} \delta_{I_{a b},\left\langle B_{1}, B_{n}\right\rangle}
$$

Now the approximate growth of $N_{2}$ with $n$ is $N_{2} \sim k^{|n|}$ so this distribution can be approximated as

$$
\begin{aligned}
d \mu(I) & =\sum_{n \geq 1} \delta_{I, k^{n}}+\delta_{I,-k^{n}} \\
& \sim d n\left(\delta\left(I-k^{n}\right)+\delta\left(I+k^{n}\right)\right) \\
& \sim d\left(\frac{\log |I|}{\log k}\right) \\
& \sim \frac{1}{\log k}\left(\frac{d I}{|I|}\right)
\end{aligned}
$$

where $I$ is a non-zero integer.

Now, if we have a fixed $k$ and a single sequence of world-volume theories, since the actual distribution (4.12) is sparse, one might not regard this approximation as very accurate. On the other hand, if one was considering a large collection of such theories for varying $k$, say arising by wrapping branes on different sets of cycles in different CY's, one would add a variety of distributions of this form, and generically produce a total distribution

$$
d \mu(I) \sim \frac{d I}{|I|}
$$

This illustrates the type of simplification we might gain by considering the problem as a whole. 
Note that there are an infinite number of theories, and the distribution is not normalizable. In the string theory application, the distribution is finite only with the tadpole cancellation constraint, which will set the $\mathrm{K}$ theory class of the resulting configuration. In the toy example, this could be modelled by setting $N_{1}$ and $N_{2}$, and considering theories which can be obtained by partitioning these fixed numbers among simple objects. One could also obtain representative results by placing a cutoff at $I \sim N_{\max } \sim 100$, the generic order of the Chern classes which enter this condition. This suggests the normalized distribution

$$
d \mu_{\text {norm }}(I) \sim \frac{1}{2 \log N_{\max }} \theta\left(N_{\max }-|I|\right) \frac{d I}{|I|}
$$

as the final approximate distribution for the toy model.

We now consider generalizing this to the case of $N$ nodes. We could proceed the same way in terms of the spectrum of bound states of $N$ elementary objects; quite a bit is known in simple cases and we will return to this in subsequent work. However the basic constraint which was operating in the toy example was a simplified form of Seiberg duality. While there we used the action of Seiberg duality on both the $N_{i}$ and $I_{i j}$, since the ranks of gauge groups $N_{i}$ in the end were largely constrained by anomaly cancellation, and the interesting result was for $I_{i j}$, we might try to look for a distribution $d \mu\left(I_{i j}\right)$ invariant under (3.10); in other words

$$
d \mu\left(I_{i j}\right)=d \mu\left(I_{i j}-2 I_{i n}-2 I_{n j}+I_{i n}\left|I_{n j}\right|\right)
$$

for any choice of $n$ (of course a natural solution would also be invariant under permutation on the indices and this choice would not matter). This is a linear condition on the ensemble, because each application of Seiberg duality relates one theory in the ensemble to another single theory in the ensemble 10

Thus, it is an interesting question whether there are "natural" distributions which satisfy (4.14). Note that the reason to think that the correct distribution approximately satisfies (4.14) is a bit subtle as it is not because we are describing a set of theories which are all related by Seiberg duality. Rather, it is analogous to the idea we discussed above that a randomly generated ensemble of (for example) $\mathcal{N}=4 \mathrm{SYM}$ theories should have couplings $\tau$ drawn from the $S L(2, \mathbb{Z})$-uniform distribution. After making duality identifications on the resulting space of theories, generic distributions which are generated by other means will tend to map into the simplest distributions compatible with the duality.

10 The nonlinear condition which appeared in the original version of the paper would have been appropriate for a transformation which combined different theories, but is incorrect here. 
The simplest candidate dual distribution is of course the uniform distribution with $d \mu(I)$ constant and independent of $I$. While we cannot claim to have ruled this out, it does not fit with putting bounds on the numbers of branes (tadpole cancellation), the toy model, or experience with brane construction (which tends not to produce models with large intersection numbers), so we do not consider this the preferred choice. Rather, it seems more likely to us that the correct distribution shares the general power law $d I /|I|$ behavior of the toy model, which we will refer to as "duality scaling" behavior here.

To illustrate this within the simplest possible model ensemble, we can take the components $I_{i j}$ with $i<j$ to be independently power law distributed, say as

$$
d \mu(I)=\alpha \delta_{I, 0}+\beta \sum_{n \geq 1} n^{\gamma}\left(\delta_{I, n}+\delta_{I,-n}\right) .
$$

Within the independent component approximation, the condition (4.14) is roughly

$$
d \mu(|I|) \sim d \mu\left(|I|^{2}\right)
$$

where $|I|$ is the average magnitude of a component of $I_{i j}$. Leaving aside the uniform distribution, this is best solved by $d I /|I| \sim d\left(I^{2}\right) /\left|I^{2}\right|$, so that $\gamma=-1$ is preferred by this condition.

Thus, we adopt as our simple model of the ensemble of quiver gauge theories arising from CY compactification, the distribution 4.15) with $\gamma=-1$, and a cutoff

$$
|I| \leq N_{\max }
$$

as in our previous discussion, leading to the (approximate) normalization condition

$$
1=\alpha+2 \beta \log N_{\max }
$$

The coefficients should be $O(1)$ and we will simply take $\alpha=1 / 2$, so that $\beta=$ $1 / 4 \log N_{\max } \sim 1 / 18$ for $N_{\max } \sim 100$.

This leads to a unit normalized ensemble, representing the fraction of quiver theories which are expected to have a particular chiral matter spectrum. This should be multiplied by the total number of quiver theories to obtain an ensemble of theories. In the toy model, this number was $c=\log N_{\max } / \log k$; more generally it seems plausible that this number has the general form of our previous estimates, perhaps $c^{K}$ or $c^{N_{\max }}$. As we discussed in section 3, brane-flux dualities suggest that the total number of inequivalent choices of 
brane and flux is comparable to the total number of choices of flux, and we will make our estimate in section 5 under this assumption.

While this "duality scaling" ensemble illustrates some properties of the true ensemble of quiver gauge theories which come out of brane constructions on $\mathrm{CY}_{3}$, it is probably too simple to be very realistic. In particular, the assumption of complete independence between intersection numbers is probably false. For example, one might think that a group of branes wrapped on cycles obtained by resolving a single isolated singularity, would be likely to have zero intersection numbers with other branes.

A second model ensemble which at least qualitatively reflects this structure would be to take $K \times K$ intersection matrices which are block diagonal and are direct sums of intersection matrices of dimension $K_{i}$ distributed according to the previous "duality scaling" ensemble. Such a direct sum is labelled by a partition of $K$ into positive integers $K_{i}$; we sum over these with equal weight (normalized to 1 ) to define the "partitioned duality scaling ensemble."

\subsection{A comment on the metric dependence}

All of the ensembles we discussed depend on an explicit choice of the Kähler metric on configuration space. This is appropriate as much of the physics depends on this metric, as does the very problem of finding vacua in supergravity.

Although we know the Kähler metric in a few cases, for example Ilb complex structure moduli space in the weak coupling limit, and can get interesting results this way, our ability to compute more general Kähler metrics is very limited and probably will remain so for a long time.

One can try to look for results which do not depend on this choice, such as the index formula (3.5) on a compact configuration space. So far, it seems hard to come up with interesting examples.

One can try to define ensembles which integrate over Kähler metrics. An example would be to take a reference metric $\omega_{K_{0}}$ (say the weak coupling metric) and use

$$
\int[d K] e^{-\left|\omega_{K}-\omega_{K_{0}}\right|^{2}}
$$

appropriate measures and geodesic distances are given in the math literature. As it stands, this looks like a rather poorly posed functional integral in high dimensional quantum gravity, and we would not recommend it. It might be interesting to integrate over a finite basis of variations to test universality claims. 
We suspect that progress on this perplexing point will come more indirectly. First, one can read formulas like (3.5) backwards, and argue that if we can get independent information about numbers and distribution of vacua (e.g. by duality), we can infer properties of the Kähler metric. Second, one can hope that an "exact solution" for the superpotential and so forth will pick out a mathematically natural configuration space $\mathcal{C}$ extending the ones we understand now into the strong coupling regime, and that this space will have natural candidate metrics. For now, it seems we must try to work with what we have.

\section{Estimates for the number of Standard Models}

In this section, we attempt to use the ideas we discussed to "estimate the number of standard models" in a particular framework. We are not yet in a position to make controlled estimates, but we will simply try to apply the various estimates for vacuum counting we discussed to illustrate the ideas, and to see whether or not there is an issue of predictivity. After all, if it were obvious that there were $10^{10}$ vacua or $10^{1000}$ vacua (with a reasonably uniform distribution) which qualitatively matched the Standard Model, we would more or less answer our basic question. On the other hand, if numbers $10^{100}-10^{400}$ came out, we need to consider this question in more detail. We also want to see the general shape of the estimates and which factors might dominate.

We discussed how to precisely define the "number of vacua" of string/M theory satisfying certain qualitative properties such as a given low energy gauge group, and how to estimate it. Now if one chose one of these vacua to focus on, one could go a certain ways in computing its more detailed properties, but it is clear that at present our ability to do this is very limited. Indeed, in the picture of moduli stabilization we discussed earlier, computing detailed values of couplings seems inherently difficult.

The main idea we will use to try to go further is to claim that the ensemble of all vacua of string/M theory of a certain type realize the uniform ensemble in the space of the remaining couplings. We will not give arguments beyond what we gave in sections 3 and 4 , but it should be stressed that this is a testable assumption given any ability to compute couplings, even a statistical computation of the sort we discussed earlier. Furthermore, if we found that an actual ensemble was not uniform, we would not have to give up - rather, we could propose another candidate ensemble which could better model the true ensemble, and try to draw conclusions from that. 
The basic number characterizing our knowledge of the Standard Model is the volume in coupling space measured in some natural ensemble. We quoted a number for this in the introduction, but obviously any number involves many assumptions; let us make this a bit more precise.

The main assumption we made in the estimate we gave in the introduction was that all Standard Model couplings were distributed independently and uniformly. The assumption of independence is of course false in almost all models of physical interest. For example, in grand unified models the three gauge couplings are not independent. Another class of models might try to explain the structure of the Yukawa couplings and mass matrices.

The questions of distribution and independence of couplings come to the fore when one discusses the hierarchy and cosmological constant problems. An extreme "statistical" point of view would be that string/M theory produces an ensemble of vacua in which all of the observed scales and couplings of nature are uniformly distributed. With enough vacua, one would be likely to well approximate our world. This scenario would seem rather unpromising for any sort of predictivity, but as we discussed it has not been ruled out. Probably the best hope for ruling it out (assuming there are not too many models) would be to show that the mass gap (hierarchy) does not come out with a uniform distribution; if it had (say) a narrow Gaussian distribution centered on the Planck mass, a modestly large number of vacua would not be problematic.

A more appealing scenario is one in which the hierarchy is produced by a mechanism such as the traditional exponentially small nonperturbative effect in the hidden sector, a large extra dimension effect or otherwise. Now if one had a good approximation to the exact ensemble of string/M theory vacua, the existence of such models would probably show up as non-analyticity or even a divergence in the distribution of mass gaps near zero. This might be an interesting idea to pursue, but what we will do here is instead simply restrict attention to the subclass of models which realize supersymmetry breaking at a hierarchically small scale. This replaces the strict computation of a distribution summed over all models, with the computation of the distribution of this subclass of models.

Within the subclass of models with supersymmetry breaking at $10 \mathrm{TeV}$, and assuming a uniform distribution for the couplings, the expected probability to realize the Standard Model with an acceptably small cosmological constant, is $10^{-60-10-9-9-50} \sim 10^{-138}$. Now it is very likely that the fraction of models which implement the hierarchy in this way is larger than $10^{-100}$ and that this is by far the more likely way to realize the physics we 
observe. Unfortunately this observation still does not rule out the "purely statistical" scenario, and any systematic discussion must take into account both possibilities.

Let us proceed to estimate the number of Standard Models coming from brane constructions which realize the Standard Model gauge group by wrapping three types of branes on three cycles of distinct homology class. As has been noted by Ibanez [63], the most obvious disadvantage of this class of model is that the gauge couplings do not naturally unify: the gauge coupling for a brane wrapped on a $d$-dimensional cycle $\Sigma$ is (up to $2 \pi$ 's)

$$
\frac{1}{g_{Y M}^{2}}=\frac{\operatorname{Vol}(\Sigma)}{l_{s}^{d} g_{s}}
$$

and cycles of different homology class have no reason to have the same volume. Now the grand unification of the three gauge couplings seems to be one of the best motivated extrapolations we can make beyond observable energies, and this is certainly a discouraging observation.

On the other hand, according to the rules we are playing by here, it is a problem but only in a particular quantitative way. If the couplings had unified, we would treat not all three as independent variables, leading to a naive estimate like $(1 / 25)^{3}$ for the probability of getting it from the uniform ensemble, but instead get a single $1 / 25$ for the probability of getting the unified coupling.

Under either assumption, the true probability to match the Standard Model has an additional $10^{-5}$ or so coming from the observational accuracy of the determination of $\alpha_{2}$ and $\alpha_{3}$. If we can compute threshold corrections and all other influences on these couplings very precisely, we can further restrict attention to models which get this level of structure right.

Now, compared to the other numbers which are entering into our considerations, these are all relatively small factors. While the couplings at the GUT scale are probably the most computable numbers we can get from string/M theory, they would be expected to depend on moduli in the general way we discussed before 67, 19, so it is not completely obvious that one can hope to compute even these uniquely.

Anyways, the point we are trying to make from this discussion is that for the basic question under discussion, unnaturalness of gauge coupling unification in a class of models is not a major disadvantage of the sort that not solving the hierarchy problem would be.

11 But note that in some models, these corrections are independent of moduli. [52 
Thus, if we were to find more than $O\left(10^{138}\right)$ ways to construct the Standard Model in this framework, we would again face loss of predictivity.

From our previous discussion, one might expect the main contribution to the counting to be the number of possible choices of flux. This is probably a controllable part of the problem, so this might be good news, but we should try to check this intuition.

The qualitative features we will assume in our discussion are dynamical supersymmetry breaking, and the gauge group and chiral matter spectrum of the Standard Model. Another feature one might want to include, which we will not discuss, is the tuning away of dimension 5 operators required to get acceptable rates of proton decay. Our excuse for this will be to say that in a non-unified theory, the natural suppression of dimension 5 operators would be $1 / M_{p l}$, which would suffice.

\subsection{The conditions for Standard Model matter}

The basic structure of brane constructions of the Standard Model has been given in many works such as [63]. One realizes $S U(2) \times S U(3)$ gauge symmetry by taking a configuration with two copies of the same brane $B_{2}$ and three copies of a different $B_{3}$. To avoid adjoint matter, one takes rigid branes. One generally needs distinct branes $B_{1}$ and $B_{1}^{\prime}$ associated to "hypercharge" to get the usual structure of two Higgs doublets. All brane constructions contain many $U(1)$ gauge groups, most of which are broken or anomalous. The question of which ones remain unbroken is somewhat complicated and we will ignore this, though the need for anomaly cancellation helps in getting a Standard Model $U(1)$.

Thus, the chance to get the gauge group right on general grounds is roughly the fraction of brane configurations with rigid branes

$$
B_{1}+B_{1}^{\prime}+2 B_{2}+3 B_{3}+\text { other branes. }
$$

To get the matter right, one needs branes with particular intersection numbers:

$$
\begin{aligned}
& \left\langle B_{2}, B_{3}\right\rangle=\left\langle B_{3}, B_{1}\right\rangle=\left\langle B_{3}, B_{1}^{\prime}\right\rangle=\left\langle B_{1} \cdot B_{2}\right\rangle=3 ; \\
& \left\langle B_{1}, B_{1}^{\prime}\right\rangle=\left\langle B_{1}, B_{2}\right\rangle=0 .
\end{aligned}
$$

This includes all charged matter except the right handed electron (which must appear to get anomaly cancellation) and the Higgs doublets (which are nonchiral). Of course there are more conditions on matter, superpotential and so forth, which we will ignore here.

To do the problem right, one must work in type I or Ilb orientifold theory, and choose an orientifolding. In the class of brane constructions we discuss, a large set of orientifoldings 
just consist of identifying $\mathbb{Z}_{2}$ reflection symmetries of the quiver and projecting the fields under such a reflection combined with complex conjugation. This changes the problem and the estimates we will discuss but not in a qualitative way (since $\mathbb{Z}_{2}$ symmetries are fairly generic). Since the main point of the discussion here is not to get a precise number but rather to illustrate the ideas, we omit this part of the problem and count brane constructions in type II. 12

The data we just described is computable for each $\mathrm{CY}_{3}$, but what we will now assume is that the rigid branes $B_{i}$ and $B_{i}^{\prime}$ are the basis branes of one of the Seiberg dual theories which can arise from the CY. We must take a set which cancel anomalies; rather than find this explicitly, we will grant that this can be done by using $L=O\left(c_{2}(M)\right)$ different elementary branes, and use this as the number of gauge groups in the quiver. We can then estimate the number of theories which realize a given intersection form by appealing to our model ensembles of quiver theories from section 4 . We start with the duality scaling ensemble.

The number of subsectors that a given quiver theory contains which realize (5.1) is simply the number of ways of selecting a $4 \times 4$ submatrix of $I_{i j}$ which matches the data (5.1). Given an $L \times L$ matrix, the number of ordered choices will be $L ! /(L-4) ! \sim L^{4}$, and we have six matrix elements to match. One of these is actually fixed by $S U(3)$ anomaly cancellation; there is also an overall factor of 2 since one can flip all chiralities. The resulting fraction of models is

$$
2 d \mu(0)^{2} d \mu(3)^{3}=2 \frac{\alpha^{2} \beta^{3}}{3^{3}} \sim 3 \times 10^{-6},
$$

so a quiver gauge theory with $L$ nodes randomly chosen from our ensemble will typically realize the Standard Model in $3 \times 10^{-6} L^{4}$ different ways.

This is typically a large number, but a much more stringent condition is that the resulting candidate Standard Model does not contain exotic matter charged under the Standard Model gauge group. Besides the fact that it has not been seen, the main problem with this is that it spoils grand unification, a constraint we are not imposing, but let us anyways estimate the probability to not have such matter. If we only worry about $S U(2) \times S U(3)$, this will be

$$
d \mu(0)^{2(L-4)} \sim 4^{-(L-4)}
$$

12 Another excuse for this is that doing this right with our present strategy requires a better understanding of Seiberg duality on the orientifolded theories. 
which is a major suppression. Indeed, in explicit brane constructions, it tends to be difficult to eliminate such exotic matter. 28

We can now sum the resulting estimate over our list of $\mathrm{CY}_{3}$ 's. The number $L=c_{2}(M)$ can be computed for each, but we will just take $L \sim K \sim \chi$. This gives the estimated number of Standard Model quivers

$$
N_{S M Q \text { d.s. }}=3 \times 10^{-6} \times \sum_{K=0}^{400} K^{5} 4^{-K} \sim 10^{-2} .
$$

If we trusted our model ensemble, the fact that this estimate comes out less than 1 would mean that the Standard Model was in fact difficult to realize, because of the difficulty of eliminating exotic charged matter. Of course the approximate nature of the estimate means that solutions could well exist, but probably at low $\chi$; direct search through the low $\chi$ Calabi-Yau's would be quite interesting in this case.

As we discussed earlier, the assumption of complete independence made in the duality scaling model ensemble is probably false, because one can have groups of cycles which do not intersect with other groups. The constraint of no exotic matter would thus favor realizing the Standard Model in such a group, say with branes wrapped on cycles obtained by resolving one isolated singularity. This observation has been made in the brane construction literature, but without quantitative considerations, it is hard to know how much significance to give this constraint compared to other constraints one might try to realize.

A similar estimate for the partitioned duality scaling ensemble can be obtained by using the fraction of partitions of $K$ which contain an integer $M$, which goes as $\log K / M$, to obtain

$$
N_{S M Q \text { partitioned }}=3 \times 10^{-6} \times \sum_{K=0}^{400} K \log K \sum_{M} M^{3} 4^{-M} \sim 75 .
$$

This would certainly be an interesting estimate if true, as it suggests a sparse set of solutions scattered among the various $\mathrm{CY}_{3}$ 's. At this point we are not claiming it is reliable. Rather, our point is that one can do a much better job of characterizing the true ensemble of gauge theories realizable by brane constructions with existing techniques, and a reliable estimate would be of great value in deciding how to search through the large set of possibilities.

To continue, let us grant (5.4) as a factor in the total estimate, which is

$$
N_{S M}=75 \times N_{v a c}(\text { non } \mathrm{SM} \text { branes }) .
$$

The second factor is of the type which we discussed in section 3 and gave the generic estimate $c^{N}$ for some $c>1$. Thus it is expected to be large, but smaller than the number of vacua which would be obtained by counting all combinations of branes, not separating out some to realize the Standard Model. 


\subsection{The conditions for low energy supersymmetry breaking}

A straightforward way to approach this question is to ask for a gauge sector which dynamically breaks supersymmetry. The status of this problem is reviewed in [82]. At present there is no general classification of such models, but there are special cases which are well understood such as the $(3,2)$ model. This is quite similar to the Standard Model but with intersection numbers 1 instead of 3 . Thus we need more branes $B_{1}^{\prime \prime}+2 B_{2}^{\prime}+3 B_{3}^{\prime}$ with specified intersection numbers, and get an estimate $d \mu(1)^{3} \sim 10^{-4}$. The main difference from our previous estimate is that we will not worry about exotic matter. While exotic matter brings in the real possibility of additional flat directions which spoil supersymmetry breaking, it is also known that there are many more supersymmetry breaking theories, so some possibilities may work.

Another supersymmetry breaking mechanism more in tune with the ideas developed here is simply to observe that a flux superpotential would be expected to contain many supersymmetry breaking minima, simply on grounds of genericity. Indeed, the explicit gauge models may well be dual to this type of realization. An advantage of this point of view is that much of the issue in finding supersymmetry breaking is in showing that the moduli are stabilized in a reasonable regime after supersymmetry breaking, so one needs an approach in which this can be done. This type of question can be studied in the simplified ensembles of section 4, as we will discuss in [41,44].

At the present state of knowledge, it is difficult to do better on this problem, and we will guess that a fraction $10^{-4}$ of models contain dynamical supersymmetry breaking. One also needs to estimate the probability that the supersymmetry breaking scale comes out right (not hard if we grant the usual exponential suppression) and that this sector is coupled to the observable sector in an acceptable way (e.g. which solves the $\mu$ problem); we will also assume this is not hard, say that $O\left(10^{-3}\right)$ of the models do it.

While these particular numbers have no real significance, the basic assumption we are making is that since we do not (yet) observe the supersymmetry breaking sector, one could pass this test in many ways. Using this estimate, one obtains

$$
N_{S M+S U S Y B}=10^{-5} \times N_{v a c}(\text { non SM and SUSY branes). }
$$

\subsection{The number of models}

We now have an estimate which factors into the number of ways to realize the structure assumed in our class of models, and the number of vacua which correspond to choices in the 
hidden sector. We gave various estimates for the latter, based on considering the hidden sector as made up of branes or made up of fluxes.

A simple minded way to get to a final result is to say that since our construction separated out 12 branes as special, we have $N-12$ branes in the hidden sector, and a vacuum multiplicity of the order $c^{N-12}$. This would be multiplied by the number of choices of flux.

However, it is clearly not the case that these choices are independent; for example the geometric dualities of Gopakumar and Vafa [56] are an obvious redundancy, and there are surely many more. This is the largest "systematic uncertainty" in our counting but a point on which theoretical progress can be made.

For present purposes, let us assume that all "non Standard Model" and "non susy breaking" branes can be dualized to flux.

This is an interesting assumption because after dualizing, we are left with a very specific configuration of 12 Dirichlet branes of 6 distinct types. This is typically not what will come out of anomaly cancellation for $\mathrm{CY}_{3}$ 's with large $K=b_{1,1}$; we might instead expect $O(K)$. Thus many of the $\mathrm{CY}_{3}$ 's need not be considered at all under this assumption, as all of these brane configurations are actually redundant.

The number of $\mathrm{CY}_{3}$ 's with $b_{1,1} \sim 6$ is much smaller, say $400-1000$. We could then directly apply (5.2) and our "estimate" for the likelihood of successful supersymmetry breaking to obtain

$$
\begin{aligned}
N_{S M} & \sim 3 \times 10^{-13} \times \sum_{C Y_{3} \text { with } K \sim 6} N_{\text {flux vac }} \\
& \sim 10^{-10} \times\left\langle N_{\text {flux vac }}\right\rangle
\end{aligned}
$$

in terms of the average number of flux vacua in this class (a sum which is probably dominated by the $\mathrm{CY}_{3}$ 's with large $b_{2,1} \sim 400$ ).

If we trusted this number, the upshot would be that realizing the Standard Model with low energy supersymmetry breaking is not easy, but not so difficult considering the expected number of flux vacua. While the number of qualitatively correct models would be large, the question of how many matched the couplings would depend on the number of flux vacua and the resulting distribution of couplings.

We are not going to defend this number very strongly. But we will defend the discussion which led up to it, as illustrating a new and different way to think about "string phenomenology," and suggesting all sorts of new questions about both the elements of 
string compactification and the types of theories which might lead to observable physics, which will be interesting to explore.

We believe that reliable estimates of this type could be made without having exact results for string/M theory at all couplings, but simply with better theoretical understanding of some key points which emerged in our discussion, and a good deal of work. A consistency check which one could apply to the results would be to make estimates for a variety of dual realizations of the same family of vacua, and see if one gets rough agreement.

Not having a reliable estimate, we would still conjecture that the qualitative structure of the Standard Model is the result of discrete choices which are not that hard to realize, and that the fraction of models which meet the qualitative tests (ignoring values of couplings) is closer to $O\left(10^{-10}\right)$ than to $O\left(10^{-100}\right)$.

There is independent evidence for this, in that among the few models which have been considered in any depth, one does get Standard Model candidates or at least "nearmisses" (say with exotic charged matter). One might worry that this is a selection effect (i.e., people only study models which are likely to realize the Standard Model), but this is clearly not true, as almost none of the $\mathrm{CY}_{3}$ 's on the list of $[71]$ have been considered at all.

If this estimate is even approximately valid, this shows that the main problem is to get a good estimate for numbers of flux vacua. Although an estimate of $O\left(10^{100}\right)$ seems plausible, it is not at all ruled out that there are $\mathrm{CY}_{3}$ 's or brane gauge theories with extremely large numbers of vacua, so the question of testability remains open.

\section{Conclusions}

In this work, we proposed a new approach to the "vacuum selection problem" of string/M theory. We believe that we should not postpone work on this problem until either an "exact solution" or some key "Vacuum Selection Principle" (or both) are discovered. Rather, we should learn to work better with the many known "vacuum selection principles" (more simply, "tests") of fitting observation and other well motivated theoretical frameworks such as cosmology, by getting a rough overall picture of the set of all string/M theory vacua and estimates of how difficult it is to satisfy each of the various tests, meaning what fraction of the total number of vacua pass a test or combination of tests. We believe such estimates will be invaluable for any systematic program to test string/M theory, even as better selection principles emerge. 
Making such estimates requires working with large numbers of models in a uniform way, which is only practical if one has a systematic construction. We discussed IIb brane/flux compactification on $\mathrm{CY}_{3}$, which we believe is approaching the level of sophistication needed. We identified several theoretical points which need to be clarified to make a proper discussion, such as the scope of geometric dualities in compactifications with both branes and flux.

We then proposed to simplify and perhaps make progress on this challenging problem by looking for simpler ensembles of effective theories which well approximate the ensemble of effective theories which actually comes out of string/M theory, in the sense that the correct estimated fraction of vacua passing a particular test is reproduced by the ensemble. It is worth emphasizing that this project has meaning if and only if a "true ensemble of effective theories coming from string/M theory" exists. While this is not proven, it only depends on two essential claims - namely, that string/M theory has a precise definition in which the candidate physical vacua can be described by low energy effective theories, and that the set of these effective theories is "locally finite" (as we discussed), possibly after imposing physical restrictions such as those we discussed (e.g. the bound on compactification volume). The first claim is supported by the fact that physical observation can be fit with effective field theory, while the failure of the second would probably eliminate any sort of predictivity, and both claims are consistent with our present understanding of string/M theory, so we consider them as very reasonable hypotheses on which to base further considerations.

Granting the existence of this ensemble, the study of candidate approximations to it is a well posed problem, and well posed questions in string/M theory tend to have simple answers. To begin this study, we gave examples of simple ensembles, which have some rough similarity to the real problems coming out of string/M theory, and illustrations of the type of computations one could do with them. The basic computation one can do is to find expected numbers of supersymmetric and nonsupersymmetric vacua, and the dependence of these numbers on parameters of the ensemble. We also illustrated how dualities can be used to constrain these ensembles.

We then made some rough estimates of numbers of Standard Models which suggest that in Ilb theory, the main ingredient in getting a good estimate is to estimate the number of possible choices of flux. There are many other ingredients which need to be refined, among which the problem of whether and in what sense a "uniform" ensemble of effective theories will emerge. 
We stress that, although the specific constraints of realizing the Standard Model, supersymmetry breaking and so forth, have of course been much studied, before this work there has been no way to quantify how constraining each of these considerations might be within string theory. By considering ensembles, this can be quantified, giving us a framework in which to systematize, evaluate and combine these considerations.

Although the ensembles we considered are somewhat crude, we can progress by formulating better ones which try to reflect more of the structure of the problem, and test our hypothesized ensembles against statistics of sample sets of string vacua constructed either systematically, or by choosing random examples and doing detailed model-by-model analysis. By finding better ensembles, we will be improving our understanding of the distribution of string/M theory vacua in a relatively concrete way. One might think of the structure of a good ensemble as capturing a "stringy" concept of naturalness, which could improve on traditional ideas of naturalness in guiding string phenomenology.

Suppose we had these estimates: what would we do next? The best argument that they are worth having is that what we would do next depends very much on what comes out. Obviously one would want to focus on tests which seem difficult to meet yet are theoretically tractable; it is not a priori obvious which ones these are.

We even argued that depending on what comes out, we might find that string/M theory has much less predictive power than we thought, perhaps none. At present it is reasonable to think that string/M theory will have predictive power, but we should admit that we do not really know, and try to find out.

Acknowledgements

This work originated in the talk [39], and has benefitted from discussions with many people, particularly Bobby Acharya, Sujay Ashok, Paul Aspinwall, Tom Banks, Rafael Bousso, Philip Candelas, Mirjam Cvetic, Frederik Denef, Savas Dimopoulos, Tamar Friedmann, Steve Giddings, Mark Gross, Shamit Kachru, David Kutasov, Sheldon Katz, Alistair King, Maxim Kontsevich, Hong Liu, Juan Maldacena, Greg Moore, Lubos Motl, Miles Reid, Paul Seidel, Savdeep Sethi, Steve Shenker, Bernie Shiffman, Nati Seiberg, Richard Thomas, Sandip Trivedi, Cumrun Vafa, Edward Witten, Steve Zelditch and Chen-Gang Zhou. I would also like to acknowledge general inspiration drawn from the works of Mikhail Gromov. Finally, I thank the Banff International Research Station for providing a pleasant environment for the completion of this work.

This research was supported in part by DOE grant DE-FG02-96ER40959. 


\section{References}

[1] B. Acharya, M. Aganacic, K. Hori and C. Vafa, hep-th/0202208.

[2] B. Acharya, hep-th/0212294.

[3] B. Acharya, to appear.

[4] C. Angelantonj and A. Sagnotti, Phys. Rept. 371 (2002) 1-150; hep-th/0204089.

[5] P. S. Aspinwall, B. R. Greene, and D. R. Morrison, Nucl. Phys. B416 (1994) 414-480.

[6] P. S. Aspinwall and M. R. Douglas, JHEP 0205031 (2002); hep-th/0110071.

[7] T. Banks, hep-th/0211160.

[8] T. Banks and L.J. Dixon, Nucl. Phys. B307, 93 (1988).

[9] T. Banks, M. Dine and M. R. Douglas, hep-ph/0112059.

[10] T. Banks, M. Dine and M. Graesser, hep-ph/0210256.

[11] T. Banks, M. Dine and L. Motl, JHEP 0101 (2001) 031; hep-th/0007026.

[12] C. E. Beasley and M. R. Plesser, JHEP 0112, 001 (2001), hep-th/0109053.

[13] K. Becker and M. Becker, Nucl.Phys. B477 (1996) 155-167; hep-th/9605053.

[14] K. Becker, M. Becker, M. Haack and J. Louis, hep-th/0204254.

[15] D. Berenstein and M. R. Douglas, hep-th/0207027.

[16] P. Bleher, B. Shiffman and S. Zelditch, Comm. Math. Phys., 208 (2000), 771.

[17] R. Bousso and J. Polchinski, JHEP 0006 (2000) 006, hep-th/0004134.

[18] J. D. Brown and C. Teitelboim, Nucl. Phys. B297 (1988) 787.

[19] F. Cachazo, B. Fiol, K. A. Intriligator, S. Katz and C. Vafa, Nucl. Phys. B 628, 3 (2002), hep-th/0110028.

[20] F. Cachazo, M. R. Douglas, N. Seiberg and E. Witten, JHEP 0212 (2002) 071; hepth/0211170.

[21] F. Cachazo, N. Seiberg and E. Witten, JHEP 0302 (2003) 042; hep-th/0301006.

[22] P. Candelas, G. Horowitz, A. Strominger, and E. Witten, Nucl. Phys. B258 (1985) $46-74$.

[23] P. Candelas, X. C. de la Ossa, P. S. Green, and L. Parkes, Nucl. Phys. B359 (1991) 21.

[24] J. F. G. Cascales and A. M. Uranga, hep-th/0303024.

[25] S. Coleman and F. De Luccia, Phys. Rev. D21 (1980) 3305.

[26] D. A. Cox and S. Katz, Mirror Symmetry and Algebraic Geometry, Mathematical Surveys and Monographs 68, AMS, 1999.

[27] M. Cvetic, G. Shiu, A.M. Uranga, hep-th/0107166.

[28] M. Cvetic, private communication.

[29] K. Dasgupta, G. Rajesh and S. Sethi, JHEP 9908 (1999) 023, hep-th/9908088.

[30] F. Denef, JHEP 0008 (2000) 050, hep-th/0005049.

[31] D.-E. Diaconescu, M. R. Douglas and J. Gomis, hep-th/9712230.

[32] D.-E. Diaconescu and M. R. Douglas, hep-th/0006224. 
[33] R. Dijkgraaf and C. Vafa, hep-th/0208048.

[34] R. Y. Donagi, Asian J. Math. 1 (1997) 214-223, alg-geom/9702002.

[35] S.K. Donaldson and P.B. Kronheimer, The Geometry of Four-Manifolds, Oxford: Clarendon Press.

[36] M.R. Douglas, B. Fiol and C. Römelsberger, "The spectrum of BPS branes on a noncompact Calabi-Yau," hep-th/0003263.

[37] M.R. Douglas, lectures at the 2001 Trieste spring school, available on the web at http://www.ictp.trieste.it .

[38] M. R. Douglas, "String Compactification with $N=1$ Supersymmetry," in C. Bachas, A. Bilal, M. R. Douglas and N. A. Nekrasov, eds., Unity from Duality: Gravity, Gauge Theory and Strings, Les Houches 2001, North Holland. Not available on the web.

[39] M. R. Douglas, Lecture at JHS60, October 2001, Caltech. Available on the web at http://theory.caltech.edu.

[40] M. R. Douglas, Lecture at Strings 2002, Cambridge UK. Available on the web at http://strings.cam.ac.ul.

[41] M. R. Douglas, S. Ashok, and others, work in progress.

[42] M. R. Douglas, S. Govindarajan, T. Jayaraman, and A. Tomasiello, hep-th/0203173, to appear in Comm. Math. Phys..

[43] M. R. Douglas and G. Moore, hep-th/9603167.

[44] M. R. Douglas, B. Shiffman and S. Zelditch, work in progress.

[45] M. R. Douglas, E. Witten and others, work in progress.

[46] M. R. Douglas and C.-G. Zhou, to appear.

[47] R. Friedman, J. W. Morgan, and E. Witten, J. Alg. Geom. 8 (1999) 279-401, alggeom/9709029.

[48] W. Fulton, Intersection Theory, Springer, 1998.

[49] J. L. Feng, J. March-Russell, S. Sethi and F. Wilczek, Nucl. Phys. B602 (2001) 307; hep-th/0005276.

[50] B. Feng, A. Hanany, Y. H. He and A. M. Uranga, JHEP 0112, 035 (2001), hepth/0109063.

[51] B . Fiol, JHEP 0207 (2002) 058; hep-th/0205155.

[52] T. Friedmann and E. Witten, hep-th/0211269.

[53] D. Gepner, Nucl. Phys. B296 (1988) 757.

[54] S. B. Giddings, S. Kachru and J. Polchinski, Phys. Rev. D66 (2002) 106006; hepth/0105097.

[55] E. Gimon and J. Polchinski, Phys. Rev. D54 (1996) 1667; hep-th/9601038.

[56] R. Gopakumar and C. Vafa, Adv. Math. Theor. Phys. 3 (1999) 1415; hep-th/9811131.

[57] M.B. Green, J.H. Schwarz, E. Witten, Superstring Theory, Volume II. Cambridge: Cambridge University Press.

[58] M. B. Green and J. H. Schwarz, Phys. Lett. 149B, 117. 
[59] B. R. Greene, "String Theory on Calabi-Yau Manifolds," in C. Esthimiou and B. Greene, editors, Fields, Strings and Duality, TASI 1996, pages 543-726, World Scientific, 1997, hep-th/9702155.

[60] M. Gross, private communication.

[61] S. Gukov, C. Vafa and E. Witten, Nucl. Phys. B584 (2000) 69; hep-th/9906070.

[62] J. H. Horne and G. Moore, Nucl. Phys. B432 (1994) 109; hep-th/9403058.

[63] L. E. Ibanez, hep-ph/0109082.

[64] C. Johnson, hep-th/0007170.

[65] S. Kachru, M. Schultz and S. Trivedi, hep-th/0201028.

[66] S. Kachru, R. Kallosh, A. Linde and S. Trivedi, hep-th/0301240.

[67] V. Kaplunovsky, hep-th/9205070.

[68] S. Katz, private communication.

[69] A. D. King, Quart. J. Math. Oxford (2), 45 (1994), 515-530.

[70] S. Kobayashi, Hyperbolic Complex Spaces, Springer 1998.

[71] M. Kreuzer and H. Skarke, Adv. Theor. Math. Phys. 4 (2002) 1209; hep-th/0002240.

[72] J. Maldacena, Adv. Theor. Math. Phys. 2 (1998) 231; hep-th/9711200.

[73] G. Moore, hep-th/9807087.

[74] H. Ooguri and C. Vafa, Nucl. Phys. B641 (2002) 3; hep-th/0205297.

[75] C. Römelsberger, hep-th/0111086.

[76] J. Polchinski and A. Strominger, Phys. Lett. B388 (1996) 736-742, hep-th/9510227.

[77] J. Polchinski, "TASI Lectures on D-Branes," hep-th/9611050.

[78] J. Polchinski and M. J. Strassler, hep-th/0003136.

[79] J. Polonyi, as referenced in [10].

[80] R. Schimmrigk, alg-geom/9612012.

[81] N. Seiberg, Nucl. Phys. B 435, 129 (1995); hep-th/9411149.

[82] Y. Shadmi and Y. Shirman, Rev. Mod. Phys. 72 (2000) 25-64.

[83] A. Strominger, Nucl. Phys. B274 (1986) 253.

[84] A. Strominger and C. Vafa, Phys. Lett. B379 (1996) 99; hep-th/9601029.

[85] F. Tanaka and S. F. Edwards, J. Phys. F: Metal Phys. 10 (1980) 2769.

[86] P. K. Tripathy and S. P. Trivedi, hep-th/0301139.

[87] C. Vafa, Adv. Theor. Math. Phys. 2 (1998) 207; hep-th/9711067.

[88] C. Vafa, J. Math. Phys. 42 (2001) 2798; hep-th/0008142.

[89] J. Wess and J. Bagger, Supersymmetry and Supergravity, Princeton Univ. Press., 1990.

[90] E. Witten, Nucl. Phys. B202, 253, 1982.

[91] E. Witten, Nucl. Phys. B460 (1996) 541; hep-th/9511030.

[92] S. Zelditch, math.CA/0208104. 